\title{
Lista de CONTRIBUCIONES PUBLICADAS EN EL BOLETÍN DE LA Sociedad Botánica de MÉxICO DURANTE EL PERIODO ENERO DE 1944 - JUNIO DE 2007
}

\author{
marco Antonio Romero-Romero y Jorge A. Meave \\ Departamento de Ecología y Recursos Naturales, Facultad de Ciencias, \\ Universidad Nacional Autónoma de México, México 04510, D.F., México.
}

$\mathbf{S}^{\mathrm{s}}$ presenta la lista de todas las contribuciones incluidas a lo largo de 64 años durante los cuales ha publicado el Boletín de la Sociedad Botánica de México. Este periodo incluye desde el número 1 (enero de 1944) hasta el 80 (junio de 2007). Se compendian los títulos y los nombres de los autores exactamente como aparecieron en cada trabajo, y no necesariamente como fueron impresos en las tablas de contenidos. Algunos errores tipográficos se señalan con la palabra [sic] entre corchetes. La finalidad es ofrecer una referencia revisada de los nombres de autores y títulos de los trabajos, tal como fueron firmados originalmente. Por otra parte, con el fin de dar continuidad al trabajo de indización realizado anteriormente (ButandaCervera, 1976, 1981, 1990; Magaña-Rueda, 1997), en el próximo número ( 81 , diciembre de 2007) se publicará el índice de los trabajos aparecidos en los números 61 al 81 (1997-2007).

\section{Literatura citada}

Butanda-Cervera A. 1976. Índice del Boletín de la Sociedad Botánica de México. Nos. 1-35, 1944-1975. Con prólogo de E. Hernández-X. Sociedad Botánica de México, S.C., México, D.F.

Butanda-Cervera A. 1981. Índice acumulativo del Boletín de la Sociedad Botánica de México de los números 1 (1944) al 40 (1981). Boletín de la Sociedad Botánica de México 40:73-132.

Butanda A. 1990. Índice acumulativo del Boletín de la Sociedad Botánica de México de los números 41 (1981) al 50 (1990). Boletín de la Sociedad Botánica de México 50:167-214.

Magaña-Rueda P. 1997. Índice acumulativo del Boletín de la Sociedad Botánica de México del número 41 (1981) al número 60 (1997). Boletín de la Sociedad Botánica de México 61:105117.

\section{Boletín de la Sociedad Botánica de México}

\section{Número 1, enero 1944}

Exploración botánica de la barranca de Tolantongo

Paray, Ladislao

Investigaciones botánicas en México

Martínez, Maximino

\section{Número 2, enero 1945}

Breve relación de algunas de las principales plantas observadas en el Distrito Sur de la

Baja California

Martínez, Maximino

Notas sobre algunas orquídeas

Schwarz, Américo
2 Nombres botánicos de algunas plantas de Tepoztlán, Mor.

$7 \quad$ Reko, B. P.

Un zacate emigrante (Tricholaena rosea Nees) Itié, Gabriel

$1 \quad$ Número 3, octubre de 1945

Notas sobre las palmas de México 3

Hernández-Xolocotzi, E.

Un árbol que se extingue en la cañada de 5

15 Contreras, D.F.

Roldán, Ángel 
Notas sobre la flora del centro de Chiapas

Sharp, Aaron J.

El nogal de nuez encarcelada en el Valle de México

Roldán, Ángel

La flora maravillosa de Tepoztlán

Hernández, Porfirio

Nuevas localidades de algunas plantas

Paray, Ladislao

Número 4, mayo de 1946

Señor Dr. Phil. Carlos Reiche

Roldán, Ángel

Laelias mexicanas

Balme, Juan

Exploraciones botánicas en el norte del estado

de Puebla

Paray, Ladislao

La hierba de Quetzalcoatl

Reko, B. P.

El género Engelhardtia de Orizaba, Ver.

Miranda, $F$.

La distribución del género Podocarpus en

México (1)

Sharp, Aaron

Los géneros fanerogámicos mexicanos

Reko, B. P.

Nombres tarascos de plantas

Reko, B. P.

Número 5, mayo de 1945

Eizi Matuda y la flora de Chiapas, México

Hernández-Xolocotzi, Efraim

Los frijoles y otras leguminosas cultivadas

en Chiapas

Hernández-Xolocotzi, Efraim

Las pteridofitas en la Sierra de San Cristóbal, Chiapas

Little Jr., Elbert L.

Sobre la no existencia del cipres Cupressus

thurifera H.B.K.

Martínez, Maximino

El árbol del papel en el México antiguo

Reko, B. P.

Segundo viaje a la barranca de Tolantongo

Paray, Ladislao

Nombres botánicos del Manuscrito Badiano

Reko, B. P.

Número 6, marzo de 1948

Los Cupressus mexicanos

Martinez, Maximino

A través de la Sierra Madre Occidental

Paray, Ladislao
7 Apuntes sobre la flora de Guerrero

Reko, B. P.

9

Una sapotacea [sic] nueva para México

Reko, B. P.

13 El ciprés en la mitología mexicana

Reko, B. P.

16

\section{Número 7, octubre de 1948}

Las coníferas silvestres del Valle de México

Martínez, Maximino

1 El Chilcuague (Heliopsis longipes):

planta insecticida

$2 \quad$ Little Jr., Elbert L.

La palmera de coateco

10 Silva, Jesús

Número 8, abril de 1949

13 Exploraciones en el estado de Hidalgo

Paray, Ladislao

15 Nombres botánicos chinantecos

Reko, B. P.

17 Las pseudotsugas de México

Martínez, Maximino

Distribución del género Podocarpus en México

19 Sharp, Aaron J.

La manera de producir maíz híbrido: selección

41 de especies auto-polinizadas

Donath, H. R.

Número 9, octubre de 1949

1 Tres plantas poco conocidas de la flora mexicana Matuda, Eizi

$4 \quad$ Los ciruelos de Yucatán

Souza-Novelo, Narciso

Estudio botánico de las palmas oleaginosas

7 de México

Hernández-Xolocotzi, Efraím

El género Senecio en el Valle de México

10 Paray, Ladislao

Número 10, abril de 1950

12 Un zodíaco botánico

Reko, Blas $P$.

20 Orquídeas mexicanas

Balme, Juan

23 La jojoba

Aguirre-Benavides, Gustavo

\section{Número 11, diciembre de 1950}

1 Nota florística de una asociación importante del

7 Sharp, A. J.; Hernández-Xolocotzi, E.; Crum, Howard; Fox, William B. 
El género Lamourouxia en México

Paray, Ladislao

Notas relativas a la distribución de orquídeas

mexicanas

Nagel, Otto

El marañón

Aguirre-Benavides, Gustavo

Clave para la determinación de las tribus

mexicanas de la familia de las orquídeas

Miranda, $F$.

\section{Número 12, junio de 1951}

A checklist of mosses of northeastern Mexico Crum, Howard A.

Lista de las especies de musgos del noreste de México

Crum, H. A.

Breves notas sobre las pteridofitas de la barranca de Omitlán, Hgo.

Sánchez-Mejorada, Hernando; Chávez, Carlos

\section{Número 13, diciembre de 1951}

Algunos datos ecológicos sobre vegetación de Lerma

Herrera, Teófilo

Exploraciones en la Sierra de Juárez

Paray, Ladislao

Notas cactológicas

Bravo-H., Helia

\section{Número 14, junio de 1952}

El género Datura en México

Matuda, Eizi

Las cactáceas del Valle de México

Gold, Dudley

Los camotes del pochote (Ceiba parvifolia) de Puebla

Muller, Cornelius $H$.

Apuntes biográficos del Sr. Mateo Botteri, profesor de historia natural e idiomas en el Colegio de Estudios Preparatorios de Orizaba Langman, Ida $K$.

Dos nuevas aráceas de América Central

Matuda, E.

\section{Número 15, junio de 1953}

Las compuestas del Valle Central de México

Paray, Ladislao

Otros musgos de Tamaulipas

Crum, Howard A.; Sharp, A. J.

Hibiscus spiralis Cav. malváceas

Paray, Ladislao

Burmaniáceas de Chiapas

Matuda, Eizi
5 Nuevas dioscóreas de México

Matuda, Eizi

17 Nota bibliográfica

Langman, Ida

Número 16, febrero de 1954

Los encinos de México

Martínez, Maximino

Nuevas fanerógamas de México

Paray, Ladislao

Número 17, noviembre de 1954

1 Commelináceas nuevas de México y Belice

Matuda, Eizi

3 Las compuestas del Valle Central de México 6

Paray, Ladislao

Catálogo de las plantas silvestres y cultivadas

del jardín botánico de Tuxtla Gutiérrez, Chiapas

Palacios-Rincón, Miguel Ángel

La distribución de Fagus en México

Fox, William B.; Sharp, A. J.

Visita a Guanajuato - junio 26-28, 1954

1 Gold, Dudley B.

Cultivo del Cyclamen

Cuevas-Alemán, $B$.

Número 18, julio de 1955

11 Algunas especies nuevas de México

Matuda, Eizi

Ensayo de evaluación de las relaciones entre

los géneros Conzattia, Peltophorum y Cercidium

1 Miranda, $F$.

Algunas observaciones acerca de la vegetación de

13 la región de Escárcega, Campeche y zonas cercanas Bravo-Hollis, Helia

18 Apuntes para una clase de botánica

Hernández-Xolocotzi, Efraim

Observaciones sobre los encinos de Baja California

Carter, Annetta

Liberty Hyde Bailey: marzo 15 de 1858 - diciembre

25 de 1954

Martínez, $M$.

22 Nueva localidad de Monstera tuberculata

Matuda, E.

\section{Número 19, julio de 1956}

1 El género Eupatorium en la Valle Central de México 1 Paray, Ladislao

13 Una nueva especie de Pachycereus 16

Bravo-H., Helia

17 Dos nuevos Anthurium de Chiapas 19

Matuda, Eizi

19 Las dalias

Cuevas-Alemán, Baltasar 
El Dr. Elmer D. Merril

Martínez, Maximino

Número 20, marzo de 1957

El género Bidens en el Valle de México

Paray, Ladislao

Metasequoias cultivadas en México

Martínez, Maximino

Una especie de Veratrum en Durango

Martínez, Maximino

\section{Número 21, agosto de 1957}

Dioscóreas nuevas de México y Centroamérica

Matuda, Eizi

Dos arbustos notables del estado de Yucatán

Miranda, Faustino

Gentiana mirandae y Coropsis [sic] lucida,

sp. nov. de México

Paray, Ladislao

Algunas asociaciones vegetales de los terrenos del lago de Texcoco

Rzedowski, J.

Localidades de la Picea chihuahuana

Martínez, Maximino

Notas adicionales al género Senecio en el

Valle de México

Paray, Ladislao

Iconografía de las yucas mexicanas I

Piña-L., Ignacio

Número 22, enero de 1958

Nuevas compuestas de México

Paray, Ladislao

Estudio de las dioscóreas mexicanas

Cox, Don K.; Hernández-Corzo, Antonio;

Matuda, Eizi; González-Durán, José G.

Especies del género Boletus de la Sierra de las

Cruces y del Desierto de los Leones, D.F.

Gispert de Imaz, Monserrat

Las compuestas del Valle Central de México

Paray, Ladislao

Nota bibliográfica

Langman, Ida K.

\section{Número 23, diciembre de 1958}

Dedicatoria al Dr. Faustino Miranda

Contribución al estudio de algunas especies de Asprergilli [sic] aisladas en la República Mexicana

Riba y N. Esparza, Ramón

New Mexican grasses

Swallen, Jason $R$

Mirandaceltis, a new genus from Mexico

Sharp, A. J.
Notes on Lesquerella (Cruciferae) in Mexico

Rollins, Reed C.

Lobelia splendens Humb. \& Bonpl. ex Willd.,

A poorly understood member of the Lobelia

1 cardinals complex

McVaugh, Rogers

El género Stevia en el Valle de México y

sus alrededores

14 Matuda, Eizi

Introducción al estudio de los manglares

Cuatrecasas, José

Contribución al estudio de la vegetación y

1 de la flora en algunos lugares del norte de México Valdés-Gutiérrez, Javier

Estudio preliminar de las formas de clima en las zonas cálidas y de sus relaciones con la vegetación

15 Perrin de Brichambaut, Guy

Notas adicionales para una bibliografía sobre la vegetación de Chiapas

19 Langman, Ida K.

Combinación nueva de una gramínea mexicana Hernández-X., $E$.

\section{Número 24, noviembre de 1959}

Acción antimicrobiana de algunos antibióticos extraídos de hongos superiores

Pérez-Silva, Evangelina

Sinopsis de los conocimientos sobre los hongos alucinogenos [sic] mexicanos Guzmán-H., Gastón

Nuevas especies Anthurium de Oaxaca

$1 \quad$ Matuda, Eizi

Los Penstemon de México, I. Sobre la confusión

entre Penstemon lanceolatus y Penstemon imberbis Straw, Richard M.

Salsola kali var. tenuifolia, una peligrosa maleza exótica que está extendiéndose hacía [sic] el centro de México

Rzedowsky [sic], J.

Número 25, agosto de 1960

Estudios citológicos sobre algunas especies del género Tripsacum

Prywer-L., Czeslawa

Revisión de técnicas histológicas para el estudio

de las algas marinas macroscópicas

Sánchez-Rodríguez, Ma. Elena

10

Lista preliminar de las algas marinas del litoral del estado de Veracruz
Huerta-M., Laura

Contribución al conocimiento de la vegetación del suroeste del estado de Zacatecas (República Mexicana)

Guzmán-H., Gastón; Vela-Gálvez, Luciano 
Aprovechamiento de las algas marinas

Huerta-M., Laura

Número 26, julio de 1961

Notas sobre algunos hongos mexicanos

Welden, A. L.; Lemke, P. A.

Las primeras Pteridofitas introducidas al Jardín

Botánico de la Universidad Nacional Autónoma de México

Herrera, Teófilo

Clave de los géneros mexicanos de gramíneas

Swallen, Jason R.; Hernández-X., Efraim

Plantas nuevas del sur de México

Miranda, F.

Tres estudios botánicos en la selva Lacandona,

Chiapas, México: Introducción

Mills, Thomas $H$.

Tres estudios botánicos en la selva Lacandona,

Chiapas, México

Miranda, Faustino

Informe sobre la vegetación actual de un sitio arqueológico de la civilización Chalchihuites, y de sus alrededores, vecino a la ciudad de Durango, República Mexicana

Hendricks, Albert J.

\section{Número 27, noviembre de 1962}

The Penstemons of Mexico, II. Penstemon hartwegii, Penstemon gentianoides, and their allies Straw, Richard M.

Una nueva especie arbórea de la familia Malvaceae

Gómez-Pompa, Arturo

Una especie epifita de Yucca de la selva lacandona Gómez-Pompa, Arturo; Valdés, Javier

Notas sobre el género Philodendron (Aráceas) en México

Matuda, Eizi

Contribuciones a la fitogeografía florística e histórica de México I. Algunas consideraciones acerca del elemento endémico en la flora mexicana Rzedowski, J.

Vegetación de una corriente de lava de formación reciente, localizada en el declive meridional de la sierra de Chichinautzin

Espinosa-Garduño, Judith

\section{Número 28, septiembre de 1963}

Notas sobre la familia Loranthaceae y el parasitismo secundario

Riba, Ramón

Meiosis en Tripsacum zopilotense Hern. y Rand.

Prywer-L., Czeslawa
Notas sobre la tipificación de Pithecolobium schaffneri S. Wats.

Rzedowski, J.

El género Opsiandra en México

Gómez-Pompa, Arturo

Los tipos de vegetación de México y su clasificación

Miranda, Faustino; Hernández-X., Efraim

\section{Número 29, julio de 1965}

Sistemática, histología y ecología de los hongos

el género Helvella del Valle de México

Gómez, Palmira; Herrera, Teófilo

120 Estudio sobre algunos Tremellales de México

Lowy, $B$.

133 Estudios acerca de árboles y arbustos de América

Chromosome numbers in some Mexican grasses

177 flora of Popocatépetl and Iztaccíhuatl

La vegetación de México

Relaciones geográficas y posibles orígenes de la

1 Alteraciones inducidas por los rayos $\mathrm{X}$ en los

Número 30, mayo de 1969

Faustino Miranda (1905-1964)

La ceremonia de homenaje al Dr. Faustino

El Dr. Faustino Miranda, como hombre y

Recuerdo de Faustino Miranda

El Dr. Faustino Miranda como investigador botánico 10

El Dr. Faustino Miranda como maestro

$1 \quad$ Maximino Martínez (1888-1964)

El profesor Maximino Martínez $\quad 24$

Recuerdos del profesor Maximino Martínez tropical, principalmente de México

Miranda, Faustino

Gould, Frank W.

A preliminary ecological study of the alpine

Beaman, John H.

Gómez-Pompa, Arturo flora de México

Rzedowski, $J$. cromosomas de las células meristemáticas de la raíz de Vicia faba I. Aspectos técnicos Villalobos-Pietrini, Rafael

Gómez-Pompa, Arturo Miranda, efectuada el 19 de febrero de 1965

Valdéz-G., Javier

como amigo

Ruiz-Oronoz, Manuel

del Pozo, Efrén C.

Hernández-X., Efraim

Gómez-Pompa, Arturo

Rzedowski, Jerzy

Miranda, Faustino 
El género Monarda en México

Scora, Rainer $W$.

Algunos métodos automáticos para la

elaboración de etiquetas de herbario

Scheinvar, Léia; Gómez-Pompa, Arturo

El proyecto Index Nominum Genericorum

Langman, Ida K.; Southwell, Mary F. F.

Influencia de las aves en la vegetación de la

Laguna de Majahual en los Tuxtlas, Ver.

Sousa-S., Mario

Citología y distribución de Bouteloua

karwinskii y B. chasei

Reeder, John R.; Reeder, Charlotte G.

Las gramíneas dioicas de México

Reeder, John R.

Literatura para las briofitas de México

Delgadillo M., Claudio

El itinerario y las colectas de Sessé y Mociño

en México

McVaugh, Rogers

\section{Número 31, diciembre de 1970}

Estudios sobre los poliporáceas de Nuevo León,

II. Observaciones sobre las especies conocidas y discusiones acerca de su distribución en México

Castillo, José; Guzmán, Gastón

Estudios sistemáticos del género Microspermum

(Compositae)

Rzedowski, Jerzy

Hallazgo de una especie nueva de Draba (Cnuciferae)

en el Valle de México

Calderón de Rzedowski, Graciela

Estudio florístico-ecológico de las algas marinas de

la costa del Golfo de Tehuantepec, México

Huerta, Laura M.; Tirado-L., Jorge

Datos sobre el género Poronia (Pyrenomycetes)

en México

Pérez-Silva, Evangelina

\section{Número 32, diciembre de 1972}

Marine algae from the 1969 cruise of "Makrele" to the northern part of the Gulf of California

Norris, James $N$.

Macromicetos mexicanos en el herbario The

National Fungus Collections de E.U.A.

Guzmán, Gastón

La confusión de tipificación y de nomenclatura de dos especies americanas del género Sesbania (Leguminosae): $S$. cavanillesii y $S$. drumondii

Bunting, G.S.; Ingram, John

Una nueva Frankeniaceae del norte de México

González-Medrano, Francisco
31 Dos especies nuevas del género Microspermum

(Compositae) del estado de Jalisco (México)

73 Rzedowski, Jerzy

Número 33, enero de 1974

95

Sobre la utilización del microscopio electrónico de barrido en el estudio de hojas fósiles del Cretácico

97 inferior de Patagonia, Argentina

Archangelsky, Sergio

Los registros más antiguos del polen de

113 Nothofagus (Fagaceae) de Patagonia (Argentina

y Chile)

Archangelsky, Sergio; Romero, Edgardo

121 Rodofíceas dulceacuícolas de México

Sánchez-Rodríguez, Ma. Elena

127 A revision of Periptera DC. (Malvaceae)

Fryxell, Paul A.

137 Adiciones a la flora fanerogámica del Valle de

México, II

Calderón de Rzedowski, Graciela

\section{Número 34, marzo de 1975}

1 Orígenes, desarrollo histórico y estado actual de los herbarios en el mundo

Medellín-Leal, Fernando

Comentarios al tema: Orígenes, desarrollo histórico

y estado actual de los herbarios en el mundo

Dorantes-López, Jesús

Los herbarios de México, su historia y estado actual

Díaz-Luna, Carlos Luis; Villarreal, Luz María

Comentarios al tema: Los herbarios de México, su

historia, estado actual y proyección hacia el futuro

Takaki, Francisco

El papel de los herbarios en los estudios ecológicos, fitogeográficos y en la investigación sobre el aprovechamiento de los recursos naturales

Asteinza-B., Gaiska

Comentarios al tema: El papel de los herbarios en

los estudios ecológicos, fitogeográficos y en la

investigación sobre el aprovechamiento de los

1 recursos naturales

Madrigal-Sánchez, Xavier

El herbario como instrumento de trabajo, su manejo

31 y operación

Rzedowski, J.

Comentarios al tema: El herbario como instrumento

de trabajo, su manejo y operación

Riba, Ramón

Importancia de los herbarios en el manejo

y aprovechamiento de los bosques

71 Bonilla-Beas, Reyes; Vela-Gálvez, Luciano

Comentarios al tema: Importancia de los herbarios en

el manejo y aprovechamiento de los bosques

Villarreal C., Raúl
1 
La utilidad de los herbarios en los estudios tendientes a encontrar nuevas fuentes alimenticias

Guzmán, Gastón

Comentarios al tema: La utilidad de los herbarios en los estudios tendientes a encontrar nuevas fuentes alimenticias

Huerta M., Laura

El herbario como base de estudios taxonómicos, florísticos y evolutivos

Sousa-S., Mario

Comentario al tema: El herbario como base de estudios taxonómicos, florísticos y evolutivos Espinosa-Garduño, Judith

Herbario, genética y fitomejoramiento

Hernández-X., Efraim

Comentarios al tema: Herbario, genética y

fitomejoramiento

Fuentes-Fuentes, Santiago

Las investigaciones fotoquímicas y sus relaciones con los herbarios

Marroquín, Jorge S.; Domínguez, Xorge A.

Comentarios al tema: Las investigaciones fotoquímicas y sus relaciones con los herbarios Joseph-Nathan, $P$.

Función de los herbarios en los estudios paleontológicos, paleobotánicos, estratigráficos, paleoclimáticos y paleoecológicos

Rueda-Gaxiola, Jaime; Salas, Eloy

Comentarios al tema: Función de los herbarios en los estudios paleontológicos, paleobotánicos, estratigráficos, paleoclimáticos y paleoecológicos Palacios-Ch., Rodolfo

Conclusiones

Rzedowski, J.

\section{Número 35, diciembre de 1975}

Lycium isthmense (Solanaceae) nueva especie del Istmo de Tehuantepec Chiang-Cabrera, Fernando

Literatura adicional para las briofitas de México Delgadillo-M., Claudio

A new species of Galium (Rubiaceae) from San Luis Potosí (Mexico)

Dempster, Lauramay T.

Una Poinsettia (Euphorbiaceae) nueva y atractiva de Guerrero (México)

Dressler, Robert L.

Batesimalva y Meximalva, dos géneros

nuevos de malváceas Mexicanas

Fryxell, Paul A.

Tres dicotiledóneas mexicanas nuevas de posible interés ornamental

Rzedowski, Jerzy
A preliminary list of mosses collected on tree

branches in Mexico

Thornburgh, K. R.; Sharp, Aaron J.

103 Chiranthodendron pentadactylon Larreategui

(Sterculiaceae): una especie polinizada por

aves percheras

Toledo, Víctor Manuel

111 The genus Juniperus (Cupressaceae) in Mexico and Guatemala: numerical and morphological analysis Zanoni, Thomas A.; Adams, Robert P.

\section{Número 36, diciembre de 1977}

Anomalías morfológicas en los granos de polen de

Pinus pinceana

Díaz-Luna, Carlos Luis

The tropical rain forest near its northern limits in

Veracruz, México: recent and ephemeral?

Graham, Alan

Deliberations on leaf venation as supported by the Annonaceae

Klucking, Edward P.

Pollen sequence of the Tlaloqua crater

(La Malinche volcano, Tlaxcala, Mexico)

Ohngemach, Dieter

Lluvia de polen moderno en diferentes hábitats

del Valle de México

Palacios-Chávez, Rodolfo

Morfología de los granos de polen de la familia

Moraceae en México

Ramos-Zamora, D.

El bosque de Engelhardtia (Oreomunnea) mexicana en la región de La Chinantla (Oaxaca, México): una reliquia del Cenozoico Rzedowski, Jerzy; Palacios-Chávez, Rodolfo

\section{Número 37, diciembre de 1977}

Biología floral del género Cassia en la región de

Los Tuxtlas, Veracruz

Delgado-Salinas, Alfonso O.; Sousa-Sánchez, Mario

Observaciones sobre un comportamiento atípico de

Ruppia maritima L. en una laguna costera tropical González-Gutiérrez, Martha

Estudios demográficos en plantas. Astrocaryum mexicanum Liebm. 1. Estructura de las poblaciones Piñero, Daniel; Sarukhán, José; González, Enrique The genus Machaerium (Leguminosae) in Mexico Rudd, Velva E.

Número 38, diciembre de 1979

Musgos de Zacatecas, México. I.

Delgadillo-M., Claudio; Cárdenas-S., Ma. Ángeles

Una revisión del género Pavonia en México

Fryxell, Paul A. 
Vegetation and flora of the Bolsón of Cuatro

Ciénegas region, Coahuila, Mexico. I.

Pinkava, Donald J.

Enriquebeltrania, nombre nuevo para reemplazar

a Beltrania miranda [sic] (Euphorbiaceae)

Rzedowski, J.

Tephrosia woodii (Leguminosae), una nueva

especie del estado de Oaxaca, México

Téllez-Valdéz, Oswaldo

The genus Juniperus (Cupressaceae) in Mexico and Guatemala: synonymy, key, and distributions of the taxa

Zanoni, T. A.; Adams, R. P.

\section{Número 39, junio de 1980}

Biología de poblaciones silvestres y cultivadas de Phaseolus coccineus. L.: I. Relaciones plantapolinizador Búrquez, Alberto; Sarukhán-K., José

Contribución al conocimiento de la morfología de los granos de polen de los géneros más comunes de la familia Euphorbiaceae de México

Díaz-Zavaleta, Guillermo; Palacios-Chávez, Rodolfo

Notas sobre el género Bursera [sic] (Burseraceae) en Michoacán (México). I. Tres especies nuevas de los alrededores de la presa del Infiernillo, con algunos datos relativos a la región

Guevara-Fefer, F.; Rzedowski, J.

Hallazgo en México de una euforbiácea acuática originaria de Sudamérica

Lot, Antonio; Novelo, Alejandro; Cowan, Clark P.

La germinación de Panicum hirsutum Swartz: una arvense de cultivos de zonas inundables Orozco-Segovia, Alma D.L.; Vázquez-Yanes, Carlos

Vegetation and flora of the Bolsón of Cuatro

Ciénegas Region, Coahuila, México, II

(Casuarinaceae to Loasaceae)

Pinkava, Donald J.

NOTAS BOTÁNICAS

Grabowskia geniculata (Solanaceae) y su

presencia en México

Chiang-Cabrera, Fernando

Notes on Cupressus in Mexico

Zanoni, Thomas A.

\section{Número 40, septiembre de 1981}

Un siglo de la botánica en México

Rzedowski, Jerzy

Fundación y primera década de la Sociedad

Botánica de México (1941-1951)

Hernández-X., Efraím

Cronología de los principales acontecimientos

históricos de la Sociedad Botánica de México

Lot, Antonio; Carvajal, Magda
Los Congresos Mexicanos de Botánica de 1960 a 1978

Guevara, Sergio; Moreno-Casasola, Patricia

Índice acumulativo del Boletín de la Sociedad

Botánica de México de los números 1 (1944)

al 40 (1981)

Butanda-Cervera, Armando

\section{Número 41, noviembre de 1981}

Productividad primaria de tres praderas de especies tropicales: pará (Brachiaria mutica), grama amarga (Paspalum conjugatum) y pangola

(Digitaria decumbens)

Almeida-M., Ricardo

Una nueva especie de Tauschia (Umbelliferae)

del Estado de México

Calderón de Rzedowski, Graciela; Constance, Lincoln

Nueva especie de Casimiroa (Rutaceae) de la zona árida oaxaqueño-poblana

Chiang, Fernando; González-Medrano, Francisco

Adiciones a la flora arvense del Valle de México

Espinosa-García, Francisco Javier

Distribución esporangial en estróbilos de especies de Selaginella

Fraile, Ma. Eugenia; Riba, Ramón

Rzedowskia, un nuevo género de Celastraceae de México

González-Medrano, Francisco

Sobre la ecología reproductiva de Nicotiana glauca Grah: una maleza de distribución cosmopolita

Hernández, Hector Manuel

Estructura y composición de la vegetación herbácea de un bosque uniespecífico de Pinus hartwegii, I: estructura y composición florística Obieta, Ma. Cristina; Sarukhán, José

Vegetation and flora of the Bolson of Cuatro Cienegas region, Coahuila, Mexico: III. Cactaceae to Compositae

Pinkava, Donald J.

Ormosia (Leguminosae) in Mexico, including a new species from Oaxaca

Rudd, Velva E.

NOTAS BOTÁNICAS

Nuevo registro para México de Pontederia rotundifolia

Novelo-Retana, Alejandro

Rhizophora harrisonnii (Rhizophoraceae), un nuevo registro para las costas de México Rico-Gray, Victor

Nota sobre la distribución de Carpodiptera ameliae (Tiliaceae), en México

Téllez-Valdéz, Oswaldo 
Nota aclaratoria sobre las hojas de Valeriana

vaginata (Valerianaceae)

Vega, Rosa Ma.; Rzedowski, J.

\section{Número 42, agosto de 1982}

Componentes fenológicos del sistemadecruzamiento monoico de Cnidoscolus spinosus (Euphorbiaceae) en Jalisco

Bullock, Stephen $H$.

The impact of forest thinning on microclimate in monarch butterfly (Danaus plexippus L.)

overwintering areas of Mexico

Calvert, William H.; Zuchowski, Willow; Brower,

Lincoln P.

Comparative photosynthetic characteristics of coastal and desert plants of California Mooney, Harold A.; Berry, J.; Bjorkman, O.; Ehleringer, J.

Jatropha chamelensis (Euphorbiaceae), nueva especie de la costa de Jalisco, México

Pérez-Jiménez, L. Alfredo

Estudio de los canales resiníferos de la corteza de Bursera copallifera y Bursera grandifolia Suárez-Ramos, Guadalupe; Engelman, E. Mark

The altitudinal distribution of the genus Pinus in the western United States and Mexico Yeaton, Richard I.

\section{Número 43, diciembre de 1982}

Una nueva especie de Decazyx (Rutaceae) de Tabasco y Veracruz

Chiang, Fernando

Nuevas combinaciones y una nueva variedad de Lycium (Solanaceae) de Norteamérica Chiang, Fernando

Estudios cromosómicos en Lycium (Solanaceae) de Norteamérica

Chiang, Fernando

Estudio del polen de Phaseolus chiapasanus Piper (Leguminosae, Phaseolinae)

Delgado-Salinas, Alfonso; Martínez-Hernández, Enrique; Fernández-Ortuño, Pilar

Musgos de la Península de Yucatán, México II

Delgadillo, Claudio; Cárdenas, Ángeles

Mimosa martindelcampoi Medrano (Leguminosae), una especie nueva del sudoeste de Tamaulipas, México

González-Medrano, Francisco

Aspectos ecológicos de Mimosa biuncifera y

Mimosa monancistra en el noroeste del

estado de Guanajuato

Grether, Rosaura

Neoeplingia Ramamoorthy, Hiriart \& Medrano

(Labiatae) un nuevo género de Hidalgo, México
Ramamoorthy, T. P.; Hiriart-Valencia, Patricia;

González-Medrano, Francisco

Acacia velvae y A. mirandae (Leguminosae),

dos nuevas especies para México

Rico-Arce, Ma. de Lourdes

1

Estudios quimiotaxonómicos de Bursera

(Burseraceae). I. Bursera chemapodicta sp. N.

Rzedowski, Jerzy; Ortiz, Evangelina

NOTAS BOTÁNICAS

11 Un aparato sencillo para medir la dureza del

follaje en estudios de herbivoría

Dirzo, Rodolfo; Femández-Bremauntz, Adrian; Gómez-

Rodríguez, Gabriela; González-Kladiano, Veronica

El banco de esporas de helechos en el suelo de

19 Los Tuxtlas, Ver

Pérez-García, Blanca; Orozco-Segovia, Alma;

Riba, Ramón

RESEÑA DE LIBROS

35 Flora of Chiapas. Part 2. Pteridophytes (Smith,

Alan R. Ed.)

Riba, Ramón

41 Ecología de la estación experimental Zoquiapan

(Descripción general, vegetación y fauna), (Blanco,

Z.S. et al. Eds.)

55 Pérez-Garcñia, Martha.

Plantas medicinales del estado de Yucatán

(Mendieta, R.S. y del Amo, R Eds.)

Orozco-Segovia, Alma

Estudios ecológicos en el agrosistema cafetalero

(Jiménez-Avila, E y Gómez-Pompa, A, Eds.)

Chehaibar-Nader, Tere

Tipos de vegetación y su distribución en el

estado de Tabasco y Norte de Chiapas

(Mendoza, R.L. Ed.)

Orozco-Segovia, Alma

9

Número 44, diciembre de 1983

Los géneros de Convolvulaceae en México

Austin, D. F.; Pedraza, R. A.

Nuevos registros de ciperáceas para la flora del

Valle de México y de la República Mexicana

González-E., Ma. Socorro

Selaginella rzedowskii, una nueva especie de

selaginela heterófila del Estado de Guerrero, México

Lorea-Hernández, Francisco G.

El polen del género Agave para la Península de

Yucatán

Ludlow-Wiechers, Beatriz; Ojeda, Lina

Helechos de Veracruz: Adiantum (Pteridaceae)

Palacios-Ríos, Mónica; Riba, Ramón

Análisis de la interacción entre radiaciones gamma y mms sobre la plántula de semillas de Triticale

61 pretratadas con ácido ascórbico Palomino-Hasbach, Guadalupe 
Dos nuevas especies mexicanas de la familia

Rubiaceae

Rzedowski, Jerzy

Plantae Uxpanapae I. Colubrina johnstonii

sp. nov. (Rhamnaceae)

Wendt, Tom

NOTAS BOTÁNICAS

Sobre el significado del "co" en co-evolución

Dirzo, Rodolfo

Nota sobre tres especies de ciperáceas del Valle de Mexico

González-E., Socorro

Notas sobre la fitogeografía del bosque mesófilo

de montaña en la Sierra Madre del Sur, México

Lorenzo S. A., Lila; Ramírez-Roa, Angélica;

Soto-Arenas, Miguel Ángel; Breceda, A; Calderón,

M. del C.; Cortéz, H.; Puchet, C.; Ramírez, M;

Villalón, R; Zapata, E.

RESEÑA DE LIBROS

Ferns and allied plants with special reference to tropical america (Tryon, R.M. y Tryon, A.F., Eds)

Riba, Ramón

Colonización y destrucción de bosques en

Panamá (Moreno, H. y McKay, A. Eds)

Chehaibar-Nader, Tere

El peregrinar de las flores mexicanas (Mociño,

J.M. y Losada Eds.)

Pérez-Nasser, Nidia

\section{Número 45, diciembre de 1983}

Detalles ultraestructurales de la madera de algunas Boraginaceae de México

Barajas-Morales, Josefina

Notas taxonómicas y observaciones sobre algunas especies mexicanas de Gnaphalium (Compositae)

Espinosa-García, Francisco Javier

Vegetación insular de la península de Yucatán

Flores-Guido, José Salvador

Algunos aspectos ecológicos y fitogeográficos

de las especies de Cyperaceae en el Valle de

México. I Afinidades ecológicas

González-E., Socorro; Rzedowski, Jerzy

Análisis de la vegetación arbórea en la provincia

florística de Tehuacán-Cuicatlán

Jaramillo-Luque, Víctor; González-Medrano, Francisco

First record of Elaeagia (Rubiaceae) in Mexico, with description of a new species, E. uxpanapensis

Lorence, David H.

Estudio demográfico del lirio acuático Eichhornia crassipes (Mart) Solms: dinámica de crecimiento en dos localidades selectas de México

Niño-Sulkowska, Martha S.; Lot, Antonio
Esclarecimiento taxonómico de Nymphaea gracilis

Zucc., planta acuática endémica de México

Novelo-R., Alejandro; Lot-H, Antonio

81 Algunas adiciones al género Acourtia (Compositae -

Mutisieae)

Rzedowski, Jerzy

Dos especies nuevas de Compositae - Astereae del

$91 \quad$ Valle de México

Rzedowski, Jerzy

95 Características palinológicas de las araliáceas de México

Sosa, Victoria

97 Plantae Uxpanapae II: novedades en Violaceae y Scrophulariaceae

Wendt, Tom

NOTAS BOTÁNICAS

El número cromosómico de Grabowskia geniculata (Solanaceae)

Chiang, Fernando

103 Condalia velutina (Rhamnaceae), una planta

muy poco colectada

Fernández-Nava, Rafael

104 Registros nuevos de plantas acuáticas mexicanas

I: Myriophyllum quitense HBK (Haloragaceae)

Novelo-Retana, Alejandro

105 Especies CAM en la selva húmeda tropical de Los Tuxtlas, Veracruz

Tinoco-Ojanguren, Clara; Vázquez-Yanes, Carlos

Número 46, junio de 1984

3 Biología floral de poblaciones silvestres y cultivadas 3 de Phaseolus coccineus L. II. Sistemas reproductivos Búrquez, Alberto; Sarukhán, José

Musgos de Zacatecas, México. II

Cárdenas-S., Ángeles; Delgadillo-M., Claudio

Rango de hospedantes de Rhynchosporium secalis Carvajal-Moreno, Magda

Nuevos registros de Juncaceae y Liliaceae en el Valle de México

Galván-Villanueva, Raquel

Algunos aspectos ecológicos y fitogeográficos de las especies de Cyperaceae en el Valle de

México. II. Análisis fitogeográfico

González-E., Socorro; Rzedowski, J.

Una especie nueva de Stegnosperma

(Phytolaccaceae) del suroeste de Puebla, México González-Medrano, Francisco;

Medina-Lemos, Rosalinda

71

Una nueva especie de Scutellaria (Labiatae) de Hidalgo, México

Hiriart-Valencia, Patricia

Un género nuevo de la parte meridional de

México: Gypsacanthus (Acanthaceae,

\section{5} .

1

7 
Justicieae, Odontoneminae)

Lott, Emily J.; Jaramillo-L., Víctor; Rzedowski, Jerzy

Interpretaciones sobre la lluvia de polen en la región 53 de San Luis Potosí, México

Lozano-García, Socorro

La vegetación acuática vascular de seis lagos-cráter del estado de Puebla, México

Ramírez-García, Pedro; Novelo-R., Alejandro

NOTAS BOTÁNICAS

Nota sobre la ortografía correcta de Casimiroa

calderonii Chiang et Medrano

Chiang, Fernando

Registros nuevos de plantas acuáticas mexicanas.

II Luziola subintegra Swallen y L. spruceana

Benth. ex Doell. (Gramineae)

Novelo-R., Alejandro

RESEÑA DE LIBRO

Malezas de Buenavista, Coahuila

(Villarreal-Q., José A.)

Espinosa-García, Francisco J.

\section{Número 47, julio de 1987}

Tigridia martinezii, una especie nueva de iridáceas del estado de Hidalgo (México)

Calderón de Rzedowski, Graciela

Musgos alpinos del Nevado de Colima, México

Delgadillo-M., Claudio

Musgos de Zacatecas, México III. Síntesis y

fitogeografía

Delgadillo-M., Claudio; Cárdenas-S., Ángeles

Notas sobre la ecología reproductiva de árboles en un bosque mesófilo de montaña en Michoacán,

México

Hernández, Héctor M.; Carreón-Abud, Yasmín

New taxa and a new name in Mexican and

Central American Randia (Rubiaceae, Gardenieae)

Lorence, David H.; Dwyer, John D.

New taxa in Mexican Psychotria (Rubiaceae,

Psychotrieae)

Lorence, David H.; Dwyer, John D.

Clave genérica para las compuestas de la cuenca del río Balsas

Villaseñor, José Luis

NOTAS BOTÁNICAS

Nota sobre la identidad del segundo Tzonpotónic mencionado por Francisco Hernández en su

Historia Natural de la Nueva España

Espinosa-García, Francisco J.

Nota sobre la distribución de Savia sessiliflora

(Euphorbiaceae) en México

Lott, Emily J.
Lectotipificación de Limnocharis laforestii

Duchass. ex Griseb. (Limnocharitaceae)

Novelo-R., Alejandro

RESEÑA DE LIBRO

Flora fanerogámica del Valle de México. Vol. II.

Dicotyledoneae (Euphorbiaceae-Compositae).

(Rzedowski, Jerzy y C. de Rzedowski,

Graciela, Eds.).

Villaseñor, José Luis

\section{Número 48, diciembre de 1988}

Distribución de Rhynchosporium secalis en el

mundo y en México

Carvajal-Moreno, Magda

Algunos musgos de Tabasco, México

Delgadillo-M., Claudio; Zamudio, Sergio

Nuevos registros y nuevas combinaciones de

gramíneas del Valle de México

Herrera-A., Yolanda

Allosidastrum, un nuevo género de Malvaceae de los neotrópicos

Krapovickas, Antonio; Fryxell, Paul A.;

3 Bates, David M.

Reconocimiento florístico y consideraciones

fitogeográficas del bosque mesófilo de

7 montaña de Teocelo, Veracruz

Luna, Isolda; Almeida, Lucía; Villers, Lourdes;

13 Lorenzo, Lila

Métodos computarizados y algunas de sus

aplicaciones al estudio de la flora de Mexico

Moreno, Nancy P.; Allkin, Robert

Estudios citogenéticos de dos especies y una variedad del género Nyctocereus (Cactaceae)

Palomino-Hasbach, Guadalupe; Zuleta-Lechuga,

Socorro; Scheinvar, Leia

Inventario florístico en el cerro Tetzcotzinco,

Texcoco, Estado de México

Pulido, Ma. Teresa P.; Koch, Stephen D.

Estudio biosistemático de Eragrostis mexicana, E. neomexicana, E. orcuttiana y E. virescens

(Gramineae: Chloridoideae)

Sánchez-Vega, Isidoro; Koch, Stephen D.

Water contents of wood of tropical deciduous

forest species during the dry season

Schulze, Ernst-Detlef; Mooney, Harold A.; Bullock,

Steve H.; Mendoza, Ana

El maguey (Agave, subgénero Agave) en el altiplano potosino-zacatecano

Tello-Balderas, J. Jesús; García-Moya, Edmundo

Spigelia longiflora (Mart. et Gal.): actividad

antialimentaria en tres especies de insectos fitófagos Villavicencio, Manuel Ángel; Pérez-Escandón, Blanca E. 


\section{NOTAS BOTÁNICAS}

Notas sobre las perturbaciones naturales en un bosque mesófilo de montaña en Tamaulipas Arriaga, Laura

Nuevo registro para México de Gleditsia

(Leguminosae)

Briones-V., Oscar L.

El número cromosómico de Lycium leiospermum

I. M. Johnston (Solanaceae)

Chiang, Fernando; Viveros, Raul; Mercado, Pedro

Reducción en la fecundidad en Manfreda

brachystachya (Cav.) Rose, una agavácea

polinizada por murciélagos: los riesgos

de la especialización en la polinización

Eguiarte, Luis E.; Búrquez, Alberto

Nota sobre la identidad del tepescohuite en México

Grether, Rosaura

Consideraciones sobre la exina en Yucca, observada al microscopio de luz y microscopio de barrido

Palacios-Chávez, Rodolfo; Ludlow-Wiechers, Beatriz; Ojeda, Lina

Una adición al género Ormosia (Leguminosae)

en México: $O$. panamensis

Rudd, Velva E.; Wendt, Tom

RESEÑA DE LIBRO

Bibliografía comentada sobre pteridófitas de México. (Ramón Riba y Armando Butanda, Eds.) Arreguín-Sánchez, María de la Luz

Número 49, diciembre de 1989

La fisiología ecológica de plantas

Vázquez-Yanes, Carlos

Nutrición mineral

Rincón, Emmanuel; Huante, Pilar

Aspectos ecofisiológicos de las micorriza

Valdés, María

El papel del microclima en la fisiología ecológica vegetal

Barradas, Víctor L.

Fotosíntesis y economía del carbono en plantas superiores

Castellanos-V., Alejandro E.

El almacenamiento de semillas en la conservación de especies vegetales. Problemas y aplicaciones Vázquez-Yanes, Carlos; Toledo, Jorge R.

Fisiología y ecología del fitocromo: su función en las semillas

Orozco-Segovia, Alma

Papel de los aleloquímicos en el manejo de los recursos naturales

Anaya-Lang, Ana Luisa

Número 50, diciembre de 1990

Anatomía de la madera de algunas especies del género Mimosa (Leguminosae)

139 Chehaibar, Teresa; Grether, Rosaura

Lista florística de las algas marinas bentónicas de la costa norte de Michoacán, México

143 Dreckmann, Kurt M.; Pedroche, Francisco F.; Sentíes-G., Abel

La familia Rubiaceae en la región de Los Tuxtlas,

145 Veracruz, México

Lorence, David H.; Ibarra-Manríquez, Guillermo

Parasitismo floral por abejas sociales

147 (Meliponinae, Apidae) en el árbol quiropterófilo

Crescentia alata (Bignoniaceae)

Martínez del Río, Carlos; Bullock, Stephen H.

Estudios cromosómicos en seis especies mexicanas de commelináceas

151 Palomino-Hasbach, Guadalupe; Vázquez-Benítez, Balbina; Martínez-Almeraya, Patricia;

Mercado-Ruaro, Pedro

La familia Rhodomelaceae (Ceramiales, Rhodophyta)

en la costa del estado de Michoacán, México

Sentíes-G., Abel; Pedroche, Francisco F.;

155 Dreckmann, Kurt $M$.

Caracterización ficoflorística de los paredones de

la Sierra de Juárez, Oaxaca. Importancia de las formas de crecimiento algales en la tipificación

159 de un ambiente

Tavera-Sierra, Rosa Luz; González-González, Jorge

Fitogeografía del Valle de Tehuacán-Cuicatlán

Villaseñor, José Luis; Dávila, Patricia;

Chiang, Fernando

3 NOTAS BOTÁNICAS

La preservación con alcohol de ejemplares

colectados para herbario

Calzada, Juan Ismael; Perales-Rivera, Hugo

19 Nota sobre la propagación y pérdida de viabilidad de las semillas de Chiranthodendron pentadactylon

31 Larr. (Sterculiaceae)

García-Franco, José Guadalupe;

Perales-Rivera, Hugo R.

41 RESEÑA DE LIBROS

Contribuciones de Eizi Matuda (1894-1978)

161

al conocimiento de la flora de México

61 Riba, Ramón

Pteridophyte flora of Oaxaca, Mexico

Riba, Ramón

A neotropical companion: an introduction to the animals. Plants and ecosystems of the New

World tropics

Dirzo, Rodolfo

Índice acumulativo del Boletín de la Sociedad

Botánica de México de los números 41 (1981)

al 50 (1990)

Butanda, Armando 


\section{Número 51, agosto de 1991}

Productividad primaria neta en una selva tropical húmeda

Álvarez Sánchez, Javier

Efecto del deshierbe sobre la transmisión de la escaldadura de la cebada, por el hongo

Rhynchosporium secalis (Oud.) Davis y en la germinación de semillas

Carvajal-Moreno, Magda; Riojas-Guadiana,

Enrique; Méndez, Ignacio

Anatomía comparada de óvulos y semillas de dos especies del género Mammillaria (Cactaceae) Hernández-García, Griselda; García-Villanueva, Eduardo

Estudio morfológico de diásporas de algunas especies de plantas acuáticas del valle de México Olvera, Martha; Lot, Antonio

Anatomía del fruto de Casimiroa edulis (Rutaceae), "zapote blanco", durante su desarrollo Zavaleta-Mancera, Hilda A raceli; Engleman, E.Mark

Anatomía de la semilla de Casimiroa edulis (Rutaceae), "zapote blanco", durante su desarrollo Zavaleta-Mancera, Hilda Araceli; Engleman, E.Mark NOTAS BOTÁNICAS

Identificación de la madera de varias esculturas de origen olmeca

Barajas-Morales, Josefina

Nuevo registro de Nymphaea prolifera

(Nymphaeaceae) para México

Olvera, Martha; Lot, Antonio

RESEÑA DE LIBRO

Liquenología de México historia y bibliografía. (Godínez, José Luis y Ortega Martha M. Eds.) Butanda, Armando

\section{Número 52, diciembre de 1992}

El género Ficus, subgénero Pharmacosycea

(Moraceae) en Veracruz, México

Ibarra-Manríquez, Guillermo; Wendt, Thomas L.

Análisis sinecológico del bosque mesófilo de montaña de Omiltemi, Guerrero

Meave, Jorge; Soto, Miguel Ángel; Calvo-Irabien, Luz María; Paz-Hernández, Horacio;

Valencia-Ávalos, Susana

Transmitancia del rojo y el rojo lejano y anatomía de las hojas de algunas especies leñosas de la selva alta perennifolia

Pérez-García, Martha; Rincón, Emmanuel;

Orozco-Segovia, Alma

Morfología de los granos de polen de las familias

Aristolochiaceae, Moraceae, Phytolaccaceae y

Piperaceae del Valle de México

Quiroz-García, David Leonor; Ramos-Zamora,
Delfina; Palacios-Chávez, Rodolfo;

3 Arreguín-Sánchez, María de la Luz

Schaffneria nigripes (Aspleniaceae): morfogénesis 105 del gametofito y anatomía y morfología del esporofito

13 Riba, Ramón; Pérez-García, Blanca;

Pérez-García, Martha

NOTAS BOTÁNICAS

Tzontzápotl: Licania platypus (Chrysobalanaceae),

un recurso de potencial económico empleado

desde el México prehispánico

25 Ochoterena-Booth, Helga; Flores-Olvera, Hilda

Fisiones céntricas en cromosomas metacéntricos

de Nothoscordum bivalve (Alliaceae) de México

Palomino-Hasbach, Guadalupe; Romo, Virginia;

39 Ruenes, Rocio

RESEÑA DE LIBROS

Developmental biology of fern gametophytes

(Raghavan, Valayamghat Ed.)

Pérez-García, Blanca

Spores of Pteridophyta (Tryon, Alice F. y

67 Lugardon, Bernard Eds)

Pacheco, Leticia

\section{Número 53, diciembre de 1993}

83 Las algas de El Salto, San Luis Potosí, México:

un ejemplo de estudios florísticos en ambientes

cambiantes

93 Cantoral-Uriza, Enrique A.;

Montejano-Zurita, Gustavo

Caracterización ficológica en manantiales de la cuenca baja del sistema hidrológico del Pánuco,

$95 \quad$ México

Carmona-Jiménez, Javier; Montejano-Zurita, Gustavo

Anatomía del desarrollo de la semilla de

Hippocratea celastroides

Espinosa-Osornio, Guadalupe; Engleman, E. Mark

Mimosa bahamensis (Leguminosae) en la

Península de Yucatán, México

Grether, Rosaura; Camargo-Ricalde, Sara L.

31 Vegetación y listado florístico de la Barranca de

Acazónica, Veracruz, México

Medina-Abreo, María Elena;

Castillo-Campos, Gonzalo

Anatomía de la madera de cinco especies de

Quintana Roo, México

Rebollar, Silvia; de la Paz Pérez-Olvera,

Carmen; Quintanar, Alejandra

NOTAS BOTÁNICAS

Una pérdida irreparable para la botánica

neotropical Alwyn H. Gentry (1945-1993)

Dirzo, Rodolfo

Brian Thomas Styles, dendrólogo tropical

(1934-1993)

Germán, María Teresa; Pinzón-Picaseño, Luis M. 
Obito. Howard Scott Gentry (1908-1993)

González Medrano, Francisco

Uso de Muhlenbergia macroura (Poaceae:

Eragrostideae) en la región de Perote,

Veracruz, México

Mejía-Saules, Ma.Teresa

RESEÑA DE LIBRO

Biological diversity of Mexico: origins and distribution (Ramamoorthy, T.P.; Bye, R., Lot,

A.; Fa, J Eds.)

Chiang, Fernando

\section{Número 54, junio de 1994}

Campesinos Tének en una comunidad campesina rural de la Huasteca Potosina complementan su dieta básica con plantas locales

Ávila-Uribe, Margarita M.; Suárez-Soto, Ma. Luz;

Díaz-Perea, Francisco Javier

Anatomía de la semilla de Chenopodium berlandieri ssp. nuttalliae (Chenopodiaceae) "huauzontle" Carrillo-Ocampo, Aida; Engleman, E. Mark

The systematic status of the Agavaceae and Nolinaceae and related Asparagales in the Monocotyledons: an analysis based on the $r b c \mathrm{~L}$ gene sequence

Eguiarte, Luis E.; Duvall, Melvin R.; Learn, Jr., Gerald H.; Clegg, Michael T.

Anatomía de la semilla de cuatro especies mexicanas de Hippocratea (Celastraceae) Espinosa-Osornio, Ma. Guadalupe; Engleman, E. Mark

Iconografía inédita de la Flora Mexicana, obra de Sessé y Mociño, en el Acervo Histórico del Herbario Nacional de México, MEXU

Germán-Ramírez, María Teresa

Lista Florística de la Lacandona, Chiapas

Martínez, Esteban; Ramos-A, Clara H.;

Chiang, Fernando

Regeneración natural y diversidad de especies

arbóreas en selvas húmedas

Martínez-Ramos, Miguel

Caracteres culturales de Fomitopsis pinicola

y Heterobasidion annosum, hongos xilófagos de importancia forestal asociados a pudriciones en oyamel

Ruiz-Rodríguez, Maria Elena; Pinzón-Picaseño, Luis Manuel

Determinación palinológica del paleoambiente holocénico en la parte norte del estado de Michoacán Xelhuantzi-López, Ma. Susana

NOTAS BOTÁNICAS

Evaluación de la capacidad productiva y eficiencia de semillas para tres especies del género Pinus $(P$. montezumae Lamb., P. pseudostrobus Lind. y $P$. leiophylla Schl. \& Cham.), en la zona boscosa de
135 Nuevo San Juan Parangaricutiro, Michoacán, México Delgado-Valerio, Patricia

141 Nota sobre el polen de Sechium chinantlense Lira

\& Chiang y Parasicyos dieterleae Lira \& Torres

(Cucurbitaceae)

Lira-Saade, Rafael; Alvarado, José Luis;

Castrejón, Javier

149 RESEÑA DE LIBROS

Historia natural del Parque Ecológico Estatal

Omiltemi, Chilpancingo, Guerrero, México

(Luna-Vega, I y Llorente-Bousquets, J. Eds.)

Ramírez-Pulido, José

Studies of flora of southern Chiapas, Mexico

3 (Matuda, E. Ed.)

Oyama, Ken

Flora Mesoamericana. Vol. 6. Alismataceae a Cy peraceae (Davidse, G; Sousa, M. y Chater, A.O. Eds.)

Espejo, Adolfo
17

\section{Número 55, diciembre de 1994}

Número especial: perspectivas en las ciencias vegetales

Piñero, Daniel; Oyama, Ken

La investigación en ecología química en México

Anaya-Lang, Ana Luisa

Panorama actual de la taxonomía vegetal en México

Chiang, Fernando; Dávila, Patricia;

Villaseñor, José Luis

El conocimiento florístico de México

Dávila, Patricia; Sosa, Victoria

Un inventario biológico para México

Dirzo, Rodolfo; Raven, Peter $H$.

Las colecciones de hongos en México y su problemática en la biodiversidad del país Guzmán, Gastón

Perspectivas de la investigación en micología Herrera, Teófilo

Biología molecular en plantas

Lara-Sánchez, Miguel; Sánchez-Rodríguez, Federico

La fisiología vegetal en la botánica actual en México 53 Larqué-Saavedra, Alfonso

El Boletín de la Sociedad Botánica de México en

el contexto de las publicaciones científicas

Lot, Antonio; Butanda, Armando

Estado actual de las investigaciones etnobotánicas en México

Martínez-Alfaro, Miguel Ángel

Estudios y perspectivas sobre ecología vegetal en México

Martínez-Ramos, Miguel

Formación de recursos humanos en ciencias vegetales

Moreno-Casasola, Patricia 
Perspectivas de la investigación en fitopatología

Moreno-Martínez, Ernesto

Perspectivas de la ficología en México

Ortega, Martha M.; Godínez, José Luis

Las palmas de México: presente y futuro

Quero, Hermilo J.

Perspectivas en el estudio de las pteridofitas

Riba, Ramón; Pérez-García, Blanca

La fisiología ecológica de plantas en México

Vázquez-Yanes, Carlos

Perfil actual y perspectivas de la paleobotánica

en México

Weber, Reinhard; Cevallos-Ferriz, Sergio R.S.

\section{Número 56, junio de 1995}

ARTÍCULOS DEL 1er. SIMPOSIUM

INTERNACIONAL SOBRE AGAVACEAES [sic]

Riqueza de las familias Agavaceae y Nolinaceae en México

García-Mendoza, Abisaí; Galván-V., Raquel

Palinología de Agavaceae, una contribución biosistemática

Ojeda-Revah, Lina; Ludlow-Wiechers, Beatríz

Hutchinson (Agavales) vs. Huber y Dahlgren

(Asparagales): análisis moleculares sobre filogenia y evolución de la familia Agavaceae sensu Hutchinson dentro de las monocotiledóneas

Eguiarte, Luis E.

Análisis cladístico de la familia Agavaceae

Hernández-Sandoval, Luis

Systematics of Dasylirion: Taxonomy and molecular phylogeny

Bogler, David J.

Systematics and character evolution of the genus

Yucca L. (Agavaceae): Evidence from morphology and molecular analyses

Clary, Karen H.; Simpson, Beryl B.

ARTÍCULOS REGULARES

Acervos históricos del Herbario Nacional de

México (MEXU): documental y bibliográfico

Butanda, Armando

Estudio florístico de la porción central del municipio de San Jerónimo Coatlán, distrito de Miahuatlán (Oaxaca)

Campos-Villanueva, Álvaro; Villaseñor, José Luis

Ecología de poblaciones de plantas en una selva húmeda de México

Martínez-Ramos, Miguel; Álvarez-Buylla, Elena

\section{Número 57, diciembre de 1995 \\ ARTÍCULOS DEL 1er. SIMPOSIO INTERNACIONAL SOBRE AGAVÁCEAS \\ (Continuación) \\ Fijación de $\mathrm{CO}_{2}$ en Agave angustifolia Haw.}

José-Jacinto, Rocio; García-Moya, Edmundo

The effects of constant temperatures, light and seed

quality on the germination characteristics of

Agave americana

Pritchard, Hugh W.; Miller, Angela P.

La agroindustria del agave tequilero Agave

tequilana Weber

Valenzuela-Zapata, Ana G.

Conservación in-situ y ex-situ de las agaváceas y nolináceas mexicanas

Franco-Martínez, Irma Sonia

Los agaves de las Antillas

Álvarez de Zayas, Alberto

ARTÍCULOS REGULARES

Riqueza de especies y endemismo del componente arbóreo de la Península de Yucatán, México

Ibarra-Manríquez, Guillermo; Villaseñor, José Luis;

Durán-García, Rafael

Palinología de las lianas de la Estación de

Biología Tropical Los Tuxtlas

Lozano-García, Socorro; Ibarra-Manríquez,

Guillermo; Sosa-Nájera, Susana

Anatomía sistemática de la familia Anacardiaceae

en México. I. La corteza de Tapirira Aublet.

Terrazas, Teresa

NOTAS BOTÁNICAS

Plantas leñosas raras del bosque mesófilo de montaña. I. Fagus mexicana Martínez (Fagaceae) López-Mata, Lauro; Cházaro-Basáñez, Miguel

Una nueva localidad para la familia

Lacandoniaceae y nuevos registros para la

Reserva de Montes Azules, Chiapas, México

Reyes-García, Alberto; Sousa-S., Mario

Tipos de fruto de Yucca filifera y formas

asociadas de Tegeticula yuccasella (Lepidoptera)

Villavicencio, Miguel Ángel; Pérez-Escandón,

Blanca Estela

\section{Número 58, agosto 1996}

BOTÁNICA ESTRUCTURAL

Anatomía de la madera de ocho especies de angiospermas de clima templado

Quintanar, Alejandra; Pérez-Olvera, Carmen de la Paz; de la Cruz-Laina, Isabel;

Razo-Balcazar, Daría

\section{ECOLOGÍA}

Métodos de análisis de datos en la germinación de

semillas, un ejemplo: Manfreda brachystachya

González-Zertuche, Lourdes; Orozco-Segovia, Alma

\section{FLORÍSTICA}

Flora vascular de la Sierra Monte Grande, municipio de Charcas, San Luis Potosí, México Reyes-Agüero, J. Antonio; González-Medrano, Francisco; García-Pérez, José D. 
SISTEMÁTICA

Epistemología de la investigación taxonómica:

inferencias filogenéticas y su evaluación

de Luna, Efraín

ARTÍCULO DE REVISIÓN

Revisión sobre la alelopatía de Eucalyptus L'Herit.

Espinosa-García, Francisco J.

CONFERENCIA MAGISTRAL

Adopción de árboles nativos valiosos para la restauración ecológica y la reforestación

Vázquez-Yanes, Carlos; Batis, Ana Irene

PERSPECTIVAS

La vegetación de México: una visión histórica

Cevallos-Ferríz, Sergio R.S.;

Lozano-García, Socorro

Palaeocorología de angiospermas de la flora

mexicana durante el Mesozoico y Terciario.

Algunas evidencias palinológicas

Martínez-Hernández, Enrique;

Ramírez-Arriaga, Elia

Aspectos de la historia de la vegetación de México con base en macrofósiles de angiospermas

Cevallos-Ferriz, Sergio R.S.; Silva-Pineda, Alicia

La vegetación del Cuaternario tardío en el centro

de México: registros palinológicos e

implicaciones paleoclimáticas

Lozano-García, María del Socorro

Algunos aspectos de la evolución de la vegetación de México

González-Medrano, Francisco

Diferentes tasas evolutivas entre grupos de

angiospermas. Eudicotiledóneas

Magallón-Puebla, Susana

NOTA BOTÁNICA

Especies del género Mimosa (Leguminosae)

presentes en México

Grether, Rosaura; Camargo-Ricalde, Sara Lucía;

Martínez-Bernal, Angélica

Número 59, diciembre de 1996

BIOLOGÍA MOLECULAR

Caracterización preliminar de una secuencia

homóloga del gen LEAFY en gimnospermas

(Pinus radiata Lemm.)

Izquierdo, Liz Y.; Tapia, Rosalinda; Vergara,

Francisco; Álvarez-Buylla, Elena

BOTÁNICA ESTRUCTURAL

Anatomía del tallo y de la raíz de dos especies de

Wilcoxia Britton \& Rose (Cactaceae) del

noreste de México

Loza-Cornejo, Sofía; Terrazas, Teresa

Embriología de Erythroxylum havanense

Jacq. (Erythroxylaceae)
Vázquez-Santana, Sonia; Domínguez, César A.;

Márquez-Guzmán, Judith

ECOLOGÍA

Tipos de vegetación y diversidad $\beta$ en el Valle de

Zapotitlán de las Salinas, Puebla, México

55 Osorio-Beristain, Oscar; Valiente-Banuet, Alfonso;

Dávila, Patricia; Medina, Rosalinda

ETNOBOTÁNICA

75 Observaciones sobre un sistema de producción agrícola en el Pico de Orizaba, Veracruz, México

Ávila-Bello, Carlos $\mathrm{H}$.

MANEJO DE RECURSOS

85 La problemática socioambiental e institucional de la reforestación en la Región de la Montaña, Guerrero, México

87 Cervantes, Virginia; Arriaga, Vicente; Carabias, Julia SISTEMÁTICA

Géneros de Theaceae: aspectos taxonómicos

y nomenclaturales

Luna, Isolda; Villaseñor, José Luis

99 PERSPECTIVAS

Sistemática filogenética: teoría, análisis y datos

González, Dolores; de Luna, Efraín

113 Phylogenetic analysis of large molecular data sets

Soltis, Pamela S.; Soltis, Douglas E.

Codificación de las inserciones-deleciones en el análisis filogenético de secuencias génicas

González, Dolores

El concepto de homología filogenética y la

selección de caracteres taxonómicos

137 de Luna, Efrain; Mishler, Brent D.

NOTAS BOTÁNICAS

Psilotum complanatum Sw. en el Pedregal de San Ángel

149 Galindo-Rosete, Carlos; López-Toledo, Leonel

Plantas leñosas raras del bosque mesófilo de

montaña II. Ticodendron incognitum

Gómez-Laurito \& Gómez P. (Ticodendraceae)

Meave, Jorge; Gallardo, Claudia; Rincón, Armando

Número 60, junio de 1997

7

ECOLOGÍA

Germinación de Chiranthodendron pentadactylon

Larr. (Sterculiaceae), en respuesta a la

escarificación, temperatura y luz

Osuna-Fernández, Reyna; Laguna-Hernández,

Guillermo; Brechú-Franco, Alicia;

13 Orozco-Segovia, Alma

ETNOBOTÁNICA

Diagnóstico del conocimiento etnobotánico actual de 15

las plantas medicinales de la Península de Yucatán

FLORÍSTICA

Estructura y composición florística del Cerrado 
en el Parque Nacional Noel Kempff Mercado,

Santa Cruz, Bolivia

Mostacedo-C., Bonifacio; Killeen, Thimoty J.

SISTEMÁTICA

Sistemática filogenética y el concepto de especie

Mishler, Brent D.; de Luna, Efraín

ARTÍCULO DE REVISIÓN

Biología de las plantas carnívoras: aspectos

ecológicos y evolutivos

Alcalá, Raúl E.; Domínguez, César A.

PERSPECTIVAS

El uso de marcadores moleculares en las ciencias

vegetales

Oyama, Ken

Molecular markers

Simpson, June

Isoenzimas

Pérez-Nasser, Nidia; Piñero, Daniel

El uso de los RAPDs como marcadores moleculares en plantas

Otero-Arnaiz, Adriana; de la Cruz, Marlene;

Oyama, Ken

Amplified fragment length polymorphisms (AFLP's) 119

Simpson, June

Sistemática molecular: comparación entre

diferentes métodos y sus aplicaciones

Martínez, Mahinda

El uso de secuencias génicas para estudios

taxonómicos

González, Dolores

La región de los ITS del ADN ribosomal del

núcleo (nrADN), fuente de caracteres moleculares

en la sistemática de las gimnospermas

Quijada, Adrián; Méndez-Cárdenas, Guadalupe;

Ortiz-García, Sol; Álvarez-Buylla, Elena R.

$\mathrm{El}$ análisis filogenético: métodos, problemas y

perspectivas

Eguiarte, Luis E.; Souza, Valeria; Núñez-Farfán,

Juan; Hernández-Baños, Blanca

\section{Número 61, diciembre 1997}

BOTÁNICA ESTRUCTURAL

Ontogenia de la semilla de Yucca periculosa

(Agavaceae)

García-Villanueva, Eduardo; Engleman, E. Mark

ECOLOGÍA

Estudio de hongos micorrizógenos arbusculares

bajo árboles remanentes en un pastizal tropical

Ramírez-Gerardo, Marithza; Álvarez-Sánchez,

Javier; Guadarrama-Chávez, Patricia;

Sánchez-Gallén, Irene

Influencia de los mezquites en la composición y

cobertura de la vegetación herbácea de un

agostadero semiárido del norte de Guanajuato
Cruz-Rodríguez, Juan Antonio; García-Moya,

Edmundo; Frías-Hernández, Juan Tenorio;

Montesinos-Silva, Genaro; Flores-Flores, José Luis

ARTÍCULO DE REVISIÓN

45 Manejo de la vegetación, domesticación de plantas

31

y origen de la agricultura en Mesoamérica

Casas, Alejandro; Caballero, Javier; Mapes,

59 Cristina; Zárate, Sergio

FICOLOGÍA

Diatomeas de Texcala, Puebla

Ibarra-Vázquez, Claudia; Novelo, Eberto

71 FISIOLOGÍA

Pérdida secundaria de la fotosíntesis: el caso de

las algas incoloras y de algunos parásitos

73 Gutiérrez-Cirios-Madrid, Emma Berta

FITOGEOGRAFÍA

77 Mexican mosses: a flora of transition in the Americas 79

Delgadillo M., Claudio

MORFOLOGÍA

Variación morfológica de poblaciones silvestres y

arvenses de Solanum ehrenbergii (Solanaceae)

Luna-Cavazos, Mario

NOTA BOTÁNICA

Estudio palinológico de Ficus, subgénero

Pharmacosycea (Moraceae) de Veracruz, México

Ibarra-Manríquez, Guillermo; Martínez-Hernández, Enrique

137 Plantas leñosas raras del bosque mesófilo de

montaña III. Photinia parviflora

Williams (Rosaceae)

Cuevas-Guzmán, Ramón; Sánchez-Rodríguez,

Enrique V.; Núñez-López, Nora M.

Índice acumulativo del Boletín de la Sociedad

Botánica de México del número 41 (1981)

al número 60 (1997)

Índice por número

Índice por autor

Índice analítico

Magaña-Rueda, Patricia

\section{Número 62, junio de 1998}

BOTÁNICA ESTRUCTURAL

5 Biología celular de Lacandonia schismatica. Análisis 5 por microscopia electrónica y de fuerza atómica

Jiménez-García, Luis Felipe; Reynoso-Robles, Rafael; Fragoso-Soriano, Rogelio; Agredano-Moreno,

15 Lourdes Teresa; Segura-Valdez, María de Lourdes; González-Moreno, Sergio; Ramos, Clara Hilda;

Martínez, Esteban

Anatomía de la corteza de algunas Gimnospermas

Yáñez-Espinosa, Laura; Terrazas, Teresa

21 ECOLOGÍA

Cambios en la vegetación y uso del suelo en la

Meseta P'urhepecha, el caso de Nahuatzen, 
Michoacán, México

Alarcón-Cháires, Pablo

Efecto de la transformación de la selva a pradera sobre la dinámica de los nutrientes en un ecosistema tropical estacional en México García-Oliva, Felipe; Maass, José Manuel ETNOBOTÁNICA

Los quelites de la Sierra Norte de Puebla, México: inventario y formas de preparación

Basurto-Peña, Francisco; Martínez-Alfaro,

Miguel Ángel: Villalobos-Contreras, Genoveva FISIOLOGÍA

El efecto del microambiente en la conductividad estomática de Buddleia cordata H.B.K., en la Reserva del Pedregal de San Ángel

Ramos-Vázquez, Alfredo; Barradas, Víctor L. PERSPECTIVAS

Domesticación de plantas y recursos genéticos de México

Casas, Alejandro

Uso y manejo de Ibervillea millspaughii (Cogn.)

C. Jeffrey, Melothria pendula L. y otras especies silvestres de la familia Cucurbitaceae: posibles procesos de domesticación incipiente

Lira, Rafael; Casas, Alejandro

Tendencias evolutivas en amaranto (Amaranthus spp.) bajo selección humana en México Mapes, Cristina; Basurto, Francisco; Caballero, Javier; Bye, Robert

Origen, variación y tendencias evolutivas del henequén (Agave fourcroydes Lem.)

Colunga-García-Marín, Patricia

La domesticación de Stenocereus stellatus

(Pfeiffer) Riccobono (Cactaceae)

Casas, Alejandro; Valiente-Banuet, Alfonso;

Caballero, Javier

La domesticación de Leucaena (Fabaceae,

Mimosoideae) en México

Zárate-Pedroche, Sergio

Diversidad del cocotero en México y su evaluación al amarillamiento letal

Zizumbo-Villareal, Daniel

Los parientes silvestres del chile (Capsicum spp.) como recursos genéticos

Hernández-Verdugo, Sergio; Guevara-González, Ramón G.; Rivera-Bustamante, Rafael F.;

Vázquez-Yanes, Carlos; Oyama, Ken

NOTA BOTÁNICA

Plantas leñosas raras del bosque mesófilo de montaña IV. Cyrilla racemiflora L. (Cyrillaceae) Gallardo, Claudia; Meave, Jorge; Rincón, Armando

Número 63, diciembre de 1998 CONFERENCIAS MAGISTRALES
Botany, Latin America and the Next Millenium

Raven, Peter $H$.

39 Biodiversidad, conservación y desarrollo sustentable: algunas reflexiones respecto al futuro Sarukhán, José

La sistemática de nuestro tiempo: hechos, problemas y orientaciones

49 Crisci, Jorge V.

La conservación de la biodiversidad en México: mitos y realidades

Gómez-Pompa, Arturo

Biodiversidad y conservación en América Latina: situación actual y perspectivas

Forero, Enrique

BOTÁNICA ESTRUCTURAL

Anatomía de la corteza de tres especies de leguminosas I. Origen y desarrollo

Orduño-Cruz, Andrés; Terrazas, Teresa

ECOLOGÍA

Producción de estructuras reproductivas y fenología reproductiva de cinco especies de compuestas en una comunidad xerófita

Figueroa-Castro, Dulce María; Cano-Santana,

Zenón; Camacho-Castillo, Edgar

ETNOBOTÁNICA

91 Theobroma bicolor Humb. \& Bonpl.

(Sterculiaceae, "cuapataxtle") en el Municipio de Ayutla de los Libres, Guerrero. Composición florística de los huertos y aspectos etnobotánicos

109 Rendón-Aguillar, Beatriz; González-Soto, Graciela; Oble-Delgadillo, María Isabel; Ojeda-Cornejo, Virginia; Parra-Padilla, Rosa Elvira;

129 Pérez-González, Estela; Ramírez-Hernández, Lidia FICOLOGÍA

Fitoplancton del lago Zempoala

García-Rodríguez, Judith; Tavera, Rosaluz

141 FLORÍSTICA

Florística del bosque mesófilo de montaña de

Molocotlán, Molango-Xochicoatlán, Hidalgo, México

Mayorga-Saucedo, Rafael; Luna-Vega, Isolda;

Alcántara-Ayala, Othón

OPINIÓN

171 Hacia un nuevo código de nomenclatura biológica: el biocódigo

Sosa, Victoria; Guerrero, José A.; Flores-Castorena, Álvaro; Martínez-Alvarado, Domitila; Acevedo-Rosas, Raúl; Aguilar-Ortigoza, Carlos

ARTÍCULO DE REVISIÓN

183 Genética evolutiva del proceso de domesticación en plantas

Rendón, Beatriz; Núñez-Farfán, Juan

La sistemática de cactáceas en México, breve recuento y perspectivas

Arias, Salvador 
RESEÑA DE LIBRO

Botánica económica en bosques tropicales. Principio

y métodos para su estudio y aprovechamiento

(Ricker, Martin y Douglas C., Daly Eds)

Casas, Alejandro

Número 64, junio de 1999

BOTÁNICA ESTRUCTURAL

Desarrollo del gametofito femenino de Tagetes

patula (Asteraceae)

García-Aguilar, Marcelina; Engleman, E. Mark;

Pimienta-Barrios, Eulogio

ECOLOGÍA

Ordenación de las poblaciones silvestres de pitayo y

cardón en la Cuenca de Sayula, Jalisco

Huerta-Martínez, Francisco Martín; García-Moya,

Edmundo; Flores-Flores, José Luis;

Pimienta-Barrios, Eulogio

Distribución del manglar en cuatro sistemas

lagunares en la costa de Chiapas, México

Montes-Cartas, Claudia Gabriela; Castillo-Argüero,

Silvia; López-Portillo, Jorge

ETNOBOTÁNICA

El uso de los encinos en la región de La Malinche,

Estado de Tlaxcala, México

Espejel-Rodríguez, María Mercedes A.;

Santacruz-García, Noé; Sánchez-Flores, Moisés

FITOGEOGRAFÍA

La vegetación esclerófila perennifolia del Valle

de Tehuacán, Puebla y sus similitudes con la

vegetación esclerófila de climas mediterráneos

Flores-Hernández, Noe; Valiente-Banuet, Alfonso;

Dávila, Patricia; Villaseñor, José Luis

ARTÍCULO DE REVISIÓN

Ecología y evolución de la polinización por engaño

Castillo, Reyna A.; Cordero, Carlos;

Domínguez, César A.

Síntesis del conocimiento taxonómico, origen

y domesticación del género Capsicum

Hernández-Verdugo, Sergio; Dávila-Aranda,

Patricia; Oyama, Ken

PERSPECTIVAS

Simposium sobre Anatomía Sistemática de la Madera 85

Ángeles-Álvarez, Guillermo

A view of the current status of comparative wood anatomy

Dickison, William C.

Panorama de la anatomía sistemática de la

madera en Chile

Gómez, Miguel; Salazar, Cindy, Montenegro, Gloria

Anatomía de la madera de Anacardiaceae con énfasis 103

en los géneros americanos

Terrazas, Teresa
NOTA BOTÁNICA

Distribución geográfica y ecológica del istafiate

111

(Artemisia ludoviciana Nutt. ssp. mexicana

(Willd. ex Spreng.) Keck)

Pérez-Miranda, Ramiro; Reyes-Agüero, Juan

Antonio; Aguirre-Rivera, Juan Rogelio

\section{Número 65, diciembre de 1999}

ECOLOGÍA

Composición y estructura de una nopalera bajo

situaciones contrastantes de exposición de

ladera y herbivoría

del Castillo, Rafael F.

El bosque mesófilo de la región de Puerto

Soledad (Oaxaca), México: análisis estructural

Ruiz-Jiménez, Carlos Alberto; Meave, Jorge;

Contreras-Jiménez, José Luis

Riqueza y estabilidad de los polinizadores de los cirios (Fouquieria columnaris) del desierto sonorense: un análisis comparativo

Nabhan, Gary Paul; Donovan, Jim; Buchmann, Stephen; Búrquez, Alberto

FICOLOGÍA

Los géneros Docidium, Penium, Haplotaenium,

Pleurotaenium, Tetmemorus y Triploceras

(Desmidiales, Chlorophyta) en Cuba

Martínez-Almeida, Víctor $M$.

FITOGEOGRAFÍA

41 Distribución geográfica y diversidad de los musgos

neotropicales

Delgadillo M., Claudio

PERSPECTIVAS

Ecofisiología vegetal: una herramienta para

la conservación y restauración ecológica

Barradas, Victor L.

El ambiente de la semilla en el suelo: su efecto en

la germinación y en la sobrevivencia de la plántula

González-Zertuche, Lourdes; Orozco-Segovia, Alma;

Vázquez Yanes, Carlos

Modificación del microclima con énfasis en la

conservación y la restauración ecológica

Barradas, Víctor L.

Aspectos ecofisiológicos para la conservación de ecosistemas tropicales contrastantes

Azócar, Aura; Rada, Fermín; García-Núñez, Carlos

Fuentes de agua utilizadas por las plantas desérticas

y su importancia en planes de manejo y

restauración ecológica

Squeo, Francisco A.; Olivares, Nancy; Valenzuela, Alejandra; Pollastri, Alberto; Aguirre, Evelyn; Aravena,

Ramón; Jorquera, Carmen; Ehleringer, James R.

NOTA BOTÁNICA

Propuesta de una técnica para herborizar plantas 


\author{
Gutiérrez-Salazar, José Alejandro; \\ García-Mendoza, Abisaí \\ IN MEMORIAM \\ Enriqueta García de Miranda (1928-1999) \\ Trujillo, Teresa Reyna \\ Carlos Rafael Vázquez Yanes (1945-1999) \\ Anónimo \\ Rodolfo Palacios Chávez (1929-1999) \\ Rodríguez J., Concepción; Quiroz G., D. Leonor
}

\section{Número 66, junio de 2000 \\ ECOLOGÍA}

Análisis de crecimiento de tres especies de

Caesalpinia (Leguminosae) de la selva baja

caducifolia de Chamela, Jalisco

Rincón, Emmanuel; Huante, Pilar;

Álvarez-Añorve, Mariana

Cronología reproductora de Ceratozamia

mexicana (Cycadales)

Sánchez-Tinoco, María Ydelia; Engleman,

E. Mark; Vovides, Andrew P.

Respuesta germinativa y crecimiento de plántulas de Mammillaria magnimamma (Cactaceae) bajo diferentes condiciones ambientales

Ruedas, Marcela; Valverde, Teresa;

Castillo-Argüero, Silvia

El método comparativo en ecología vegetal

Morales, Eduardo

PERSPECTIVAS

Simposio Internacional sobre A gaváceas y Nolináceas García-M, Abisaí; Eguiarte-F., Luis; Galván-V., Raquel; Hernández-S., Luis (Eds)

Morfología de semillas, germinación y desarrollo postemergente de tres especies del género

Polianthes L. (Agavaceae)

Serrano-Casas, Héctor; Solano-Camacho, Eloy;

Ocampo-López, Adelaida

Carbohydrates and flowering in Hesperalö̈

funifera (Koch) Trel. (Samandoque)

Mclaughlin, Steven P.; Williams, Ryan R.

Remoción cuticular ("mixiote") y desarrollo foliar en 73 los agaves pulqueros (Agave salmiana y A. mapisaga)

José-Jacinto, Rocío; García-Moya, Edmundo

Maguey (Agave spp.) utilization in Mesoamerican civilization: a case for precolumbian "pastoralism"

Parsons, Jeffrey R.; Darling, J. Andrew

Agaves productores de fibras duras en el estado de Oaxaca, México

Palma Cruz, Felipe de Jesús

La familia Agavaceae en el estado de Querétaro, México

Magallán- Hernández, Fabiola;

Hernández-Sandoval, Luis
Revisión taxonómica de las especies arborescentes de Furcraea (Agavaceae) en México y Guatemala García-Mendoza, Abisaí

111 Evolución de la familia Agavaceae: filogenia, biología reproductiva y genética de poblaciones

113 Eguiarte, Luis E.; Souza, Valeria; Silva-Montellano, Arturo

115 RESEÑA DE LIBROS

Phylogeography: The History and Formation of Species (Avise, John C. Ed.)

Eguiarte, Luis E.

IN MEMORIAM

$5 \quad$ Ramón Riva y Nava Esparza (1934-1999)

Pérez-García, Blanca; Pacheco, Leticia; Espejo-Serna, Adolfo; López-Ferrari, Ana Rosa Laura Huerta Múzquiz (1913-2000)

Mendoza-G., A. Catalina

Número 67, diciembre de 2000

BOTÁNICA ESTRUCTURAL

Anatomía comparada del tallo y de la raíz de las especies del género Neoevansia Marshall (Cactaceae)

Herrera-Cárdenas, Roberto; Terrazas, Teresa;

Loza-Cornejo, Sofía

ECOLOGÍA

37 Efecto de dos especies de árboles remanentes y de un pasto en la capacidad amortiguadora del $\mathrm{pH}$ del suelo en un ecosistema tropical estacional Nava-Mendoza, Maribel; Galicia, Leopoldo; García-Oliva, Felipe

La vegetación del Valle de Tehuacán-Cuicatlán Valiente-Banuet, Alfonso; Casas, Alejandro; Alcántara, Ariel; Dávila, Patricia; Flores-Hernández, Noe; Arizmendi, María del Coro; Villaseñor,

José Luis; Ortega-Ramírez, José

\section{ETNOBIOLOGÍA}

Biología y aprovechamiento de Agave lechuguilla Torrey

Reyes-Agüero, Juan Antonio; Aguirre-Rivera, Juan Rogelio; Peña-Valdivia, Cecilia Beatriz FLORÍSTICA

Análisis florístico de la vegetación secundaria derivada de la selva húmeda de montaña de Santa Cruz Tepetotutla (Oaxaca), México Romero-Romero, Marco Antonio; Castillo, Silvia; Meave, Jorge; van der Wal, Hans NOTA BOTÁNICA

Ceratozamia euryphyllidia Vázquez Torres, Sabato \& Stevenson (Zamiaceae), un nuevo registro para Oaxaca, con comentarios sobre su hábitat y distribución altitudinal 
Rivera-Hernández, Jaime Ernesto; Salas-Morales, Silvia H.; Torres-Bahena, Elizabeth

RESEÑA DE LIBRO

Cincuenta años de botánica evolutiva [Variation and evolution in plants and microorganisms: Towards a new synthesis 50 years after Stebbins (Ayala, F.L.; Fitch, W.M. y Clegg, M.T. Eds.)] Eguiarte, Luis E.

\section{Número 68, junio de 2001 \\ BOTÁNICA ESTRUCTURAL}

Embriología de Pachycereus militaris (Audot)

Hunt (Cactaceae)

Núñez-Mariel, Citlali; Engleman, E. Mark;

Márquez-Guzmán, Judith

ECOLOGÍA

Some biological aspects of the arbuscular

mycorrhizal fungi (AMF)

Camargo-Ricalde, Sara Lucía

ETNOBOTÁNICA

Especies útiles del género Mimosa

(Fabaceae-Mimosoideae) en México

Camargo-Ricalde, Sara Lucía; Grether, Rosaura;

Martínez-Bernal, Angélica; García-García,

Verónica; Barrios-del-Rosal, Saraí

FLORÍSTICA

Flora algal del río La Magdalena, Distrito Federal, México

Ramírez-Vázquez, Mónica; Beltrán-Magos, Yenny; Bojorge-García, Miriam; Carmona-Jiménez, Javier; Cantoral-Uriza, Enrique A.;

Valadez-Cruz, Francisco

Las gimnospermas de los bosques mesófilos de montaña de la Huasteca Hidalguense, México Contreras-Medina, Raúl; Luna-Vega, Isolda;

Alcántara-Ayala, Othón

TAXONOMÍA

Nueva especie del género Sicyos L. (Cucurbitaceae) para la República Mexicana

Rodríguez-Arévalo, Isela; Lira-Saade, Rafael

Una especie nueva notable de Pinguicula

(Lentibulariaceae) de los estados de Querétaro y

San Luis Potosí, México

Zamudio-Ruiz, Sergio

RESEÑA DE LIBRO

A la recherche du temps perdu. [Enfoques

contemporáneos para el estudio de la

biodiversidad. Hernández, H.M., García-Aldrete,

A.N., Álvarez, F.; Ulloa, M., Comp.]

Eguiarte, Luis E.

IN MEMORIAM

Roberto Cruz Cisneros (1940-2001)

Ma. Susana Xelhuantzi López.
Número 69, diciembre de 2001

ECOLOGÍA

Desarrollo arquitectónico de tres especies de Acacia

Echeverry-Gómez, Amparo; Vester, Henricus F.M.

Dinámica estacional de la biomasa de las raíces

finas asociada a agregados del suelo en un

ecosistema tropical estacional

García-Oliva, Felipe; Paz-Tapia, María

The paradox of mesquites (Prosopis spp.): Invading species or biodiversity enhancers?

Golubov, Jordan; Mandujano, Maria C.;

$5 \quad$ Eguiarte, Luis E.

Relación lluvia de polen-vegetación en selvas de Quintana Roo

Islebe, Gerald A.; Villanueva-Gutiérrez, Rogel;

Sánchez-Sánchez, Odilón

15 ETNOBOTÁNICA

Manejo de huertos familiares periurbanos de

San Miguel Tlaixpan, Texcoco, Estado de México

Gaytán-Ávila, Celia; Vibrans, Heike; Navarro-

Garza, Hermilio; Jiménez-Velázquez, Mercedes

FLORÍSTICA

Diversidad florística del bosque mesófilo en el norte 63 de Chiapas y su relación con México y Centroamérica Ramírez-Marcial, Neptalí

La vegetación de la Reserva Ecológica Lomas

del Seminario, Ajusco, México

González-Hidalgo, Beatriz; Orozco-Segovia, Alma;

Diego-Pérez, Nelly

La familia Scrophulariaceae en México: diversidad y distribución

Méndez Larios, Isidro; Villaseñor Ríos, José Luis

RESEÑA DE LIBRO

Hacia una etnobotánica mexicana y moderna [Plantas, cultura y sociedad: estudio sobre la relación entre seres humanos y plantas en los albores del siglo XXI (Rendón-Aguilar, B.; Rebollar-Domínguez, S.; Caballero-Nieto, J.; Martínez Alfaro, M.A. Eds.)]

Eguiarte, Luis E.

Número 70, junio de 2002

SISTEMÁTICA Y FLORÍSTICA

Morfología de plántulas de Bursera Jacq. ex L.

(Burseraceae) y sus implicaciones filogenéticas

Andrés-Hernández, Agustina Rosa;

89 Espinosa-Organista, David

El bosque mesófilo de montaña en el municipio de

Landa de Matamoros, Querétaro, México

Cartujano, Sandra; Zamudio, Sergio; Alcántara,

Othón; Luna, Isolda

Componente florístico del desierto de San Felipe,

Baja California, México

Delgadillo-Rodríguez, José; Macías-Rodríguez, Miguel Ángel 
BOTÁNICA ESTRUCTURAL

Anatomía de la semilla de Ti gridia pavonia (Inidaceae)

Carrillo-Ocampo, Aída; Engleman, E.M.

MICOLOGÍA

Nuevos registros de Pyrenomycetes (Ascomycotina) 79 en México

Medel, Rosario

NOTA BOTÁNICA

Brasenia schreberi J.F.Gmel. (Cabombaceae), un nuevo registro para Chihuahua, México

Lot, Antonio; Ramos, Francisco;

Ramírez-García, Pedro

RESEÑA DE LIBROS

Una nueva síntesis evolutiva: el regreso de la

competencia y la adaptación [The ecology of adaptive radiation. (Schluter, D. Ed.)]

Eguiarte, Luis E.; Núñez-Farfán, Juan

\section{Número 71, diciembre de 2002}

ECOLOGÍA

La vegetación de Calakmul, Campeche, México: clasificación, descripción y distribución

Martínez, Esteban; Galindo-Leal, Carlos

Dispersal, distribution and establishment of arbuscular mycorrhizal fungi: a review

Camargo-Ricalde, Sara Lucía

Modelos arquitectónicos en la flora arbórea de la

Península de Yucatán

Vester, Henricus Franciscus Maria

SISTEMÁTICA Y FLORÍSTICA

La familia Lauraceae en el sur de México:

diversidad, distribución y estado de conservación

Lorea-Hernández, Francisco G.

PALEOBOTÁNICA

El clima durante el Plioceno en la región de

Santa María Amajac, Hidalgo, México

Aguilar, Felisa J.; Velasco-de León, Ma. Patricia

IN MEMORIAM

Ingrid Christine Olmsted (1939 - 2002)

Durán, Rafael

Edward W. Greenwood (1918 -2002)

Light, Marylin; Reddock, Joyce; Hágsater, Eric;

Soto, Miguel

\section{Número 72, junio de 2003}

ECOLOGÍA

Efecto del fuego en la estructura poblacional

y la regeneración de dos especies de encinos

(Quercus liebmanii Oerst. y Quercus magnoliifolia

Née) en la región de La Montaña (Guerrero), México

Peña-Ramírez, Víctor M.; Bonfil, Consuelo

SISTEMÁTICA Y FLORÍSTICA

Flora de la costa de Oaxaca, México: Lista florística de la región de Zimatán

Salas-Morales, Silvia H.; Saynes-Vásquez,

Alfredo; Schibli, Leo

PALEOBOTÁNICA

Palinomorfos del Holoceno en la cuenca alta

del Río Lerma, Estado de México, México

Ludlow-Wiechers, Beatriz; Almeida-Leñero,

Lucía; Sugiura, Yoko

BOTÁNICA ECONÓMICA Y ETNOBOTÁNICA

Los usos no leñosos de los encinos en México

Luna-José, Azucena de Lourdes;

Montalvo-Espinosa, Linda; Rendón-Aguilar, Beatriz

A preliminary study of the taxane chemistry and

natural history of the Mexican yew, Taxus

globosa Schltdl.

Shemluck, Melvin J.; Estrada, Eduardo; Nicholson,

Robert; Brobst, Susan W.

NOTA BOTÁNICA

Observations on the propagation of Taxus

globosa Schltdl.

Nicholson, Robert; Munn, Diana Xochitl

Número 73, diciembre de 2003

ECOLOGÍA

Determinantes de la distribución de Pinus spp.

en la Altiplanicie Central de Chiapas, México

Alba-López, María Patrocinio; González-Espinosa,

Mario; Ramírez-Marcial, Neptalí;

Castillo-Santiago, Miguel Ángel

Estructura, composición florística y diversidad de

especies leñosas de un bosque mesófilo de montaña

en la Sierra de Manantlán, Jalisco

Sánchez-Rodríguez, Enrique Valente; López-Mata,

Lauro; García-Moya, Edmundo;

Cuevas-Guzmán, Ramón

Correlación morfo-específica en flores de

Palicourea padifolia (Rubiaceae)

Hernández, Angélica; Ornelas, Juan Francisco

SISTEMÁTICA Y FLORÍSTICA

Flora y vegetación de las zonas núcleo de la Reserva

de la Biosfera Mariposa Monarca, México

Cornejo-Tenorio, Guadalupe; Casas, Alejandro;

Farfán, Berenice; Villaseñor, José Luis;

Ibarra-Manríquez, Guillermo

BOTÁNICA ESTRUCTURAL

Arquitectura foliar y anatomía de la corteza y la

madera de Quercus sartorii y Q. xalopensis (Fagaceae)

Martínez-Cabrera, Dorismilda; Terrazas, Teresa;

Zavala-Chávez, Fernando

NOTA BOTÁNICA

Sedum clavifolium Rose (Crassulaceae), nuevo

registro para la flora de Michoacán, México

García-R., Ignacio 
RESEÑA DE LIBROS

Vegetación y flora del Municipio de Tenabo, Campeche (Zamora-Crescencio, Pedro, Ed.) van der Wal, Hans

\section{Número 74, junio de 2004 \\ ECOLOGÍA}

Asignación de recursos e influencia de los cotiledones en el crecimiento de plántulas de Nectandra ambigens (Blake) C.K.Allen (Lauraceae) en una selva tropical húmeda Barajas-Guzmán, Guadalupe; Álvarez-Sánchez, Javier

Análisis estructural de un bosque mesófilo de montaña en el extremo oriental de la Sierra Madre del Sur (Oaxaca), México Mejía-Domínguez, Nancy R.; Meave, Jorge A.; Ruiz-Jiménez, Carlos A.

SISTEMÁTICA Y FLORÍSTICA

Listado florístico de la Sierra de Santa Rosa, Guanajuato, México

Martínez-Cruz, Juan; Téllez-Valdés, Oswaldo

Dinámica y conservación de la flora del matorral xerófilo de la Reserva Ecológica del Pedregal de San Ángel (D.F., México) Castillo-Argüero, Silvia; Montes-Cartas, Gabriela; Romero-Romero, Marco Antonio; Martínez-Orea, Yuriana; Guadarrama-Chávez, Patricia; Sánchez-Gallén, Irene; Núñez-Castillo, Oswaldo ETNOBOTÁNICA

Análisis preliminar de la dominancia cultural de las plantas útiles en el estado de Morelos Monroy-Ortiz, Columba; Monroy, Rafael NOTA BOTÁNICA

Validation of the name Zamia elegantissima Schutzman, Vovides et Adams Schutzman, Bart; Vovides, Andrew P.; Adam, Russell S.

\section{Número 75, diciembre de 2004 ECOLOGÍA}

Aspectos ecológicos de dos cactáceas mexicanas amenazadas: implicaciones para su conservación Álvarez, Ricardo; Godinez-Álvarez, Héctor; Guzmán, Ulises; Dávila, Patricia

¿Qué sabemos de la rareza en especies vegetales? Un enfoque genético-demográfico Esparza-Olguín, Ligia Guadalupe SISTEMÁTICA Y FLORÍSTICA

Diversidad del género Quercus (Fagaceae) en México Valencia-A., Susana

Flora bromeliológica del estado de Guerrero, México: riqueza y distribución Pulido-Esparza, Valeria Angélica; López-Ferrari, Ana Rosa; Espejo-Serna, Adolfo
Los géneros de plantas vasculares de la flora

de México

Villaseñor, José Luis

NOTA BOTÁNICA

Berrya cubensis (Griseb.) M.Gómez (Tiliaceae),

nuevo registro para el estado de Oaxaca, México

Salas-Morales, Silvia H.

5 RESEÑA DE LIBROS

El decálogo de las leguminosas [Advances in Legume Systematics- Higer Level Systematics

(Bente B., Klitgaard; Bruneau, Anne, Eds.)]

Delgado-Salinas, Alfonso

IN MEMORIAM

Javier Valdés Gutiérrez (1931-2004)

Flores-Olvera, Hilda

Leo Schibli (1958-2004)

Salas-Morales, Silvia H.

Número 76, junio de 2005

ECOLOGÍA

Estimación de la riqueza de especies de Asteraceae

mediante extrapolación a partir de datos de presencia-ausencia

Villaseñor, José Luis; Maeda, Pedro; Colín-López, J. Javier; Ortiz, Enrique

Estructura, composición y diversidad de la selva baja caducifolia del Cerro Verde, Nizanda

(Oaxaca), México

Gallardo-Cruz, José A.; Meave, Jorge A.;

Pérez-García, Eduardo A.

SISTEMÁTICA Y FLORÍSTICA

Notes on the flora of the Yucatan Peninsula IV:

Marsilea vestita Hook. \& Grev. var. vestita

(Marsileaceae), a new record and some comments about the genus in the region

Duno-de-Stefano, Rodrigo; Carnevali-Fernández-Concha, Germán; Tapia-Muñoz, José Luis; Ramírez-Morillo,

Ivón M.; Hernández-Aguilar, Silvia

BOTÁNICA ESTRUCTURAL

Embriología de las estructuras reproductoras

masculinas del género Pinguicula L. (Lentibulariaceae)

Espinosa-Matías, Silvia; Zamudio, Sergio;

Márquez-Guzmán, Judith

Anatomía de la madera de las especies mexicanas de 53 Salvia sección Erythrostachys Epl. (Lamiaceae) Ceja-Romero, Jacqueline; Pérez-Olvera, Carmen de la Paz; Rivera-Tapia, Jesús

FICOLOGÍA

Distribución geográfica y afinidad por el sustrato de las algas verdes (Chlorophyceae) bénticas de las costas mexicanas del Golfo de México y Mar Caribe Garduño-Solórzano, Gloria; Godínez-Ortega, José Luis; Ortega, Martha M. 
NOTA BOTÁNICA

La producción de frutos prolíficos en Cylindropuntia leptocaulis (Cactaceae) como una alternativa ante el fracaso de la reproducción sexual Vázquez-Delfin, Erika F.; Sánchez-Serrano, Sinthia L.; Martorell-Delgado, Carlos

RESEÑA DE LIBROS

Pteridología recreativa [A natural history of ferns (Moran, Robbin C. Ed.)]

Pacheco, Leticia; Tejero-Díez, José Daniel

\section{Número 77, diciembre de 2005 \\ ECOLOGÍA}

Germinación y supervivencia de semillas de

Thrinax radiata (Arecaceae), una especie amenazada en la Península de Yucatán

Pérez, Erika; Ceballos-González, Gerardo;

Calvo-Irabién, Luz María

SISTEMÁTICA Y FLORÍSTICA

Patrones de endemismo y disyunción de los géneros de Euphorbiaceae sensu lato: un análisis panbiogeográfico

Martínez-Gordillo, Martha; Morrone, Juan J.

Análisis florístico de La Cañada, Querétaro, México Cabrera-Luna, José Alejandro;

Gómez-Sánchez, Maricela

BOTÁNICA ESTRUCTURAL

Anatomía de la madera de especies arbóreas de un bosque mesófilo de montaña: un enfoque ecológico-evolutivo Aguilar-Rodríguez, Silvia; Barajas-Morales, Josefina

Cambios anatómicos en la corteza de Parkinsonia praecox (Ruiz et Pavón) Hawkins causados por la epífita Tillandsia recurvata L. (Bromeliaceae)

Páez-Gerardo, Luis Enrique; Aguilar-Rodríguez, Silvia; Terrazas, Teresa; Huidobro-Salas,

Ma. Elena; Aguirre-León, Ernesto

BOTÁNICA ECONÓMICA Y ETNOBOTÁNICA

Microscopic analysis and histochemical observations of the medicinal root of Iostephane heterophylla (Cav.) Benth. ex Hemsl. (Asteraceae)

Sandoval, Estela; Bye, Robert A.; Ríos, Griselda; Aguilar, María Isabel

RESEÑA DE LIBROS

La enciclopedia ilustrada de las leguminosas: sistemática, filogenia, biogeografía y usos de la familia a nivel mundial [Legumes of the world (Lewis, G.; Schrire, B.; Mackinder, B.;

Lock, M. Eds.)]

Grether, Rosaura

\section{Número 78, junio de 2006} ECOLOGÍA Y EVOLUCIÓN

Evaluación de métodos no-paramétricos para la estimación de riqueza de especies de plantas leñosas en cafetales López-Gómez, Ana María; Williams-Linera, Guadalupe

Estructura y composición de la vegetación ribereña de la barranca del río Tembembe, Morelos, México Camacho-Rico, Fernando; Trejo, Irma; Bonfil, Consuelo

Origen, mantenimiento y evolución del ginodioicismo 33 Cuevas-García, Eduardo; Abarca-García, César A. SISTEMÁTICA Y FLORÍSTICA

Notes on the flora of the Yucatan Peninsula V: New records and miscellaneous notes for the family Leguminosae Duno-de-Stefano, Rodrigo; Rico-Arce, Lourdes; Martínez-Bernal, Angélica; Gutiérrez-Báez, Celso

La flora arbórea de Michoacán, México

Cué-Bär, Eva M.; Villaseñor, José Luis; ArredondoAmezcua, Libertad; Cornejo-Tenorio, Guadalupe; Ibarra-Manríquez, Guillermo

BOTÁNICA ESTRUCTURAL

Contribución al conocimiento de Peperomia

(Piperaceae): fruto y semilla

Martínez-Colín, Marco A. Engleman, E. Mark;

Koch, Stephen D.

Anatomía foliar y del pecíolo de especies del género Rhus s. str. (Anacardiaceae)

Andrés-Hernández, Agustina Rosa; Terrazas, Teresa BOTÁNICA ECONÓMICA Y ETNOBOTÁNICA

The ceremonial use of the threatened "espadaña"

cycad (Dioon merolae, Zamiaceae) by a community

of the central depression of Chiapas, Mexico

Pérez-Farrera, Miguel A.; Vovides, Andrew P.

NOTA BOTÁNICA

Primer registro en México de Tectaria

$\times$ michleriana, Tectariaceae

Mendoza-Ruiz, Aniceto; Pérez-García, Blanca;

Valdez, Ricardo

RESEÑA DE LIBROS

La buena y la mala cara de las malezas: nuevo

recuento de las plantas arvenses para el estado de Querétaro [Atlas de malezas arvenses del estado de Querétaro (Suárez-Ramos, G.; Serrano-Cárdenas, V.; Balderas-Aguilar, P.; Pelz-Marín, R.)]

Linares Mazari, Edelmira

Orquideomanía ilustrada: historia natural, uso, ecología y conservación de las orquídeas mexicanas [Las orquídeas de México (Hágsater, E.; Soto-Arenas, M.Á.; Salazar-Chávez, G.A.; Jiménez-Machorro, R.; López-Rosas, M.A.; Dressler, R.L.)]

Meave, Jorge A.

\section{Número 79, diciembre de 2006 ECOLOGÍA}

Efectividad de algunos tratamientos pre-germinativos 9 para ocho especies leñosas de la Mixteca Alta 
oaxaqueña con características relevantes para la restauración

Martínez-Pérez, Gilberto; Orozco-Segovia, Alma;

Martorell, Carlos

Estructura y composición de la vegetación de la barra costera El Mogote, Baja California Sur, México Romero-López, Blanca Estela; León-de la Luz, José Luis; Pérez-Navarro, José Juan; de la Cruz-Agüero, Gustavo

Germinación y establecimiento de plántulas de

Pterocereus gaumeri, una cactácea columnar, rara y endémica de Yucatán, México

Méndez, Martha; Dorantes, Alfredo; Dzib, Gabriel; Argáez, Jorge; Durán, Rafael

Structure and tree diversity patterns at the landscape level in a Mexican tropical deciduous forest Durán, Elvira; Meave, Jorge A.; Lott, Emily J.; Segura, Gerardo

SISTEMÁTICA Y FLORÍSTICA

Notes on the flora of the Yucatan Peninsula VI:

Capraria mexicana Moric. ex Benth. (Scrophulariaceae s.s.), new record and some comments about the genus in the region

Duno-de Stefano, Rodrigo; Fernández-Concha, Germán Carnevali; Ramírez- Morillo, Ivón M.; Tapia, José Luis; Hernández-Aguilar, Silvia; Can-Itza, Lilia Lorena; May-Pat, Filogonio

Composición y patrones de distribución geográfica 67 de la flora del bosque de oyamel del Cerro Tláloc, México Sánchez-González, Arturo; López-Mata, Lauro; Vibrans, Heike

BOTÁNICA ECONÓMICA Y ETNOBOTÁNICA

Plantas medicinales de cuatro mercados del estado de Puebla, México Martínez-Moreno, David; Alvarado-Flores, Roberto; Mendoza-Cruz, Myrna; Basurto-Peña, Francisco NOTA BOTÁNICA

Juanulloa mexicana (Solanaceae), nuevo registro en

el occidente de México

Vargas-Rodriguez, Yalma Luisa; Cházaro-Basáñez, Miguel de Jesús; Vázquez-García, José Antonio

RESEÑA DE LIBROS

Orquídeas de la enigmática cañada [Orquídeas y otras plantas nativas de la Cañada, Cuicatlán, Oaxaca, México (Salazar, G.A.; Reyes-Santiago, J.; Brachet, C.; Pérez-Crisanto, J.)]

Pérez-García, Eduardo A.

IN MEMORIAM

Luis Alejandro Novelo Retana (1951 - 2006)

Bonilla-Barbosa; Jaime Raúl

Número 80, junio de 2007 BOTÁNICA ESTRUCTURAL

Anatomía vegetativa de Ctenitis melanosticta
(Dryopteridaceae, Pteridophyta)

Hernández-Hernández, Victoria; Terrazas, Teresa;

Mehltreter, Klaus

ECOFISIOLOGÍA

21 Los isótopos estables del hidrógeno y el oxígeno

en los estudios ecofisiológicos de plantas

Reyes-García, Casandra; Andrade, José Luis

ECOLOGÍA

Herbivory damage on oak seedlings at the edge

of cloud forest fragments

Reynoso, Juan Antonio; Williams-Linera, Guadalupe

FITOMEDICINA

Plantas con actividad anti-Helicobacter pylori:

una revisión

Castillo-Juárez, Israel; Romero, Irma

SISTEMÁTICA Y FLORÍSTICA

Tillandsia borealis (Bromeliaceae), una nueva

especie del norte de México

López-Ferrari, Ana Rosa; Espejo-Serna, Adolfo

Magnolia guerrerensis (Magnoliaceae), una especie nueva del bosque mesófilo de montaña del estado de Guerrero, México

Jiménez-Ramírez, Jaime; Vega-Flores, Karla;

Cruz-Durán, Ramiro; Vázquez-García, J. Antonio

La selva baja caducifolia en una corriente de lava volcánica en el centro de Veracruz: lista florística de la flora vascular

Castillo-Campos, Gonzalo; Dávila-Aranda, Patricia Dolores; Zavala-Hurtado, José Alejandro

NOTA BOTÁNICA

Phanerophlebia macrosora (Baker) Underw.

(Dryopteridaceae), registro nuevo para el Distrito

Federal (México)

Valdez-Avila, Ricardo; Mendoza-Ruiz, Aniceto;

Pérez-García, Blanca

IN MEMORIAM

Miguel Ángel Martínez Alfaro (23 de octubre de 1942 - 9 de febrero de 2007)

García-Marín, Patricia Colunga; Mapes-Sánchez, Cristina; Basurto-Peña, Francisco

Lista de contribuciones publicadas en el Boletín de la Sociedad Botánica de México durante el periodo enero de 1944 - junio de 2007

Romero-Romero, Marco Antonio; Meave, Jorge A.

\section{Número 80 (suplemento), junio de 2006} RESTAURACIÓN ECOLÓGICA EN MÉXICO

Restauración de bosques en territorios indígenas de

Chiapas: modelos ecológicos y estrategias de acción

González-Espinosa, Mario; Ramírez-Marcial,

Neptalí; Camacho-Cruz, Angélica; Holz, Silvia C.; Rey-Benayas, José María; Parra-Vázquez, Manuel R.

Restauración adaptable en la Meseta Purépecha,

Michoacán, México: hacia un modelo de estados 
y transiciones

Lindig-Cisneros, Roberto; Blanco-García, Arnulfo;

Sáenz-Romero, Cuauhtémoc; Alvarado-Sosa, Pedro; Alejandre-Melena, Nancy

La topografía y el hidroperíodo: dos factores que condicionan la restauración de los humedales costeros Flores-Verdugo, Francisco; Moreno-Casasola, Patricia; Agraz-Hernández, Claudia Maricusa; López-Rosas, Hugo; Benítez-Pardo, Daniel; Travieso-Bello, Ana Cecilia

Establecimiento de plantas mediante el uso de micorrizas y de islas de recursos en un matorral xerófilo deteriorado

Monroy-Ata, Arcadio; Estevez-Torres, Juan;
García-Sánchez, Rosalva; Ríos-Gómez, Ramiro

Restauración de ambientes deteriorados derivados de la selva húmeda: el uso de los hongos micorrizógenos arbusculares

33 Álvarez-Sánchez, Javier; Guadarrama, Patricia; Sánchez-Gallen, Irene; Olivera, Diego

Sucesión ecológica y restauración de las selvas húmedas

Martínez-Ramos, Miguel; García-Orth, Ximena

Las políticas públicas de la restauración ambiental en México: limitantes, avances, rezagos y retos Carabias, Julia; Arriaga, Vicente; Cervantes Gutiérrez, Virginia 